\title{
Saikosaponin A-Induced Gut Microbiota Changes Attenuate Severe Acute Pancreatitis through the Activation of Keap1/Nrf2-ARE Antioxidant Signaling
}

\author{
Jing Li, ${ }^{1}$ Jinfeng Han, ${ }^{1}$ Juan Lv, ${ }^{1}$ Shiji Wang, ${ }^{1}$ Lai Qu, ${ }^{1}$ and Yanfang Jiang $\oplus^{2}$ \\ ${ }^{1}$ Department of Intensive Care Unit, The First Hospital of Jilin University, Changchun 130021, China \\ ${ }^{2}$ Genetic Diagnosis Center, The First Hospital of Jilin University, Changchun 130021, China \\ Correspondence should be addressed to Yanfang Jiang; jiangyfjl@126.com
}

Received 1 April 2020; Revised 11 July 2020; Accepted 21 July 2020; Published 2 November 2020

Academic Editor: Carlo Gabriele Tocchetti

Copyright ( 2020 Jing Li et al. This is an open access article distributed under the Creative Commons Attribution License, which permits unrestricted use, distribution, and reproduction in any medium, provided the original work is properly cited.

\begin{abstract}
Objective. Severe acute pancreatitis (SAP) is a serious and life-threatening disease associated with multiple organ failure and a high mortality rate and is accompanied by distinct oxidative stress and inflammatory responses. Saikosaponin A has strong antioxidant properties and can affect the composition of gut microbiota. We sought to determine the effects of Saikosaponin A interventions on SAP by investigating the changes of gut microbiota and related antioxidant signaling. Methods. A SAP model was established in Sprague-Dawley (SD) rats through the injection of sodium taurocholate into the biliopancreatic duct and confirmed by elevated levels of serum lipase and amylase. The model was fed a standard diet either with saline solution or with Saikosaponin A. Fecal microbiota transplantation (FMT) from Saikosaponin A-induced rats into the rat model was performed to test the effects of gut microbiota. The composition of gut microbiota was analyzed by using 16S rRNA gene sequencing. We measured apoptotic status, inflammatory biomarkers, and Keap1-Nrf2-ARE ((Kelch-like ECH-associated protein 1) nuclear factor erythroid 2-related factor 2-antioxidant response element) antioxidant signaling. Results. Saikosaponin A intervention attenuated SAP lesions and reduced the levels of serum amylase and lipase, oxidative stress, and inflammatory responses by reducing pathological scores and affecting the serum level of oxidative and inflammatory factors. Meanwhile, the expression of Keap1-Nrf2-ARE was increased. Saikosaponin A intervention improved microbiota composition by increasing the relative abundance of Lactobacillus and Prevotella species. FMT resulted in similar results as those caused by the Saikosaponin A intervention, suggesting Saikosaponin A may exert its function via the improvement of gut microbiota composition. Conclusions. Saikosaponin A-induced gut microbiota changes attenuate SAP progression in the rat model and may be a potential natural drug for adjuvant treatment of SAP. Further work is needed to clear up the points.
\end{abstract}

\section{Introduction}

Pancreatitis is a leading complication of gastrointestinal diseases, and often initiates and exacerbates systemic inflammatory responses. The mortality rate of pancreatitis is between $1.5 \%$ and $4.2 \%$ according to the previous report [1]. Pancreatitis development will lead to the release of inflammatory indicators and cytokines, which lead to intestinal barrier damage [2]. Pancreatitis increases intestinal permeability and facilitates bacterial infection, resulting in the damage of intestinal barrier [3]. Pancreatitis is often involved with lung injury $[4,5]$, liver disease $[6]$, and other organ failure $[7,8]$.
Natural products have been found to be effective in the prevention of pancreatitis risk $[9,10]$. Radix bupleuri is a common Chinese herb and shows anti-inflammatory properties in the prevention of SAP progression [11]. Saikosaponin $\mathrm{A}$ is one of the most effective components of Radix bupleuri roots $[12,13]$. The previous work indicated that Saikosapo$\operatorname{nin} \mathrm{A}$ attenuated hyperlipidemic pancreatitis in an animal model by improving lipid metabolism and preventing the release of proinflammatory cytokines via the NF-kappaB signaling [14]. However, its function on SAP remains unclear. SAP is often characterized by recurrent episodes of inflammation and loss of tissue integrity in the intestine 
[15]. Gut microbiota is thought to be important for maintaining the balance of proinflammatory cytokines, which is closely associated with SAP progression. The knowledge for gut microbiota and its metabolites on intestinal barrier function in SAP will help us to understand the mechanism of gut failure in the pathogenesis of SAP [16]. Early dysbiosis of the gut microbiota is associated with the SAP risk, and the modulation of the gut microbiota is a potential approach in the prevention of SAP development [17].

Keap1-Nrf2-ARE ((Kelch-like ECH-associated protein 1) nuclear factor erythroid 2-related factor 2-antioxidant response element) are widely reported antioxidant signaling molecules [18, 19]. Pancreatitis is usually accompanied by the increase in the oxidative stress and inflammatory responses $[20,21]$. Free radicals play an import role in the pathophysiology of SAP, and increased free radical activities and increased concentrations of lipid peroxides have been found in both SAP patients [22] and animal models [20, 23]. Xanthine oxidase (XO) [24] and nitric oxide synthase (NOS) [25] may be the individual contribution of possible sources of free radicals. Cellular oxidative stress contributes to pancreas injury and the improvement of antioxidant capacity by increasing the levels of the reduced glutathione (GSH) level, and catalase (CAT) activity will reduce the injury [26]. The enhancement of antioxidants superoxidase dismutase (SOD) and glutathione peroxidases $(\mathrm{GPx})$ and the reduction of lipid oxidation product malondialdehyde (MDA) contribute to the recovery of SAP-induced intestinal barrier injury [27]. Therefore, the improvement of the expression of antioxidant signaling molecules will be a potential approach in the therapy of pancreatitis $[28,29]$. Therefore, the aim of present work is to explore the protective effects of Saikosaponin A on SAP and delineate the underlying mechanisms of functional Saikosaponin A by investigating the composition of gut microbiota and related molecules.

\section{Materials and Methods}

2.1. Extracts of Saikosaponin A and Analysis of HPLC. Saikosaponin A standard (purity $>99 \%$ ) was bought from Sigma (St. Louis, MO, USA) and dissolved in PBS buffer $(20 \mathrm{mM}$, $\mathrm{pH} 7.4$ ) with $0.1 \%$ BSA at room temperature. Sodium taurocholate was purchased from Sigma and dissolved in $0.9 \%$ $\mathrm{NaCl}$ to final concentration $1 \mathrm{mg} / \mathrm{ml}$. All other reagents were analytical grade.

Radix bupleuri rhizomes were purchased from Shanghai State-owned Changning Pharmacy (Shanghai, China); Saikosaponin A was isolated and characterized according to a previous report with slight modification [30]. 1000-g rhizomes of Radix bupleuri were taken, crushed, sieved through a 20 -30-mesh sieve, soaked with $100 \%$ ethanol for three days, filtered, combined with the soaking solution, and evaporated under reduced pressure on a rotary evaporator to recover ethanol. The sample was concentrated to obtain the total extracts of Radix bupleuri. The extracts were suspended in water and further extracted with petroleum ether, chloroform, and ethyl acetate to obtain a petroleum ether portion, a chloroform portion, an ethyl acetate portion, and a watersoluble portion, respectively. One thousand grams of macro- porous resin $\mathrm{H} 802$ was packed into a column $(10 \times 60 \mathrm{~cm})$. After pretreatment, ethyl acetate raffinate was added $(50 \mathrm{~g}$ of the concentrated solution was dissolved in water, and insoluble matter was filtered off). After the sample loading, the column was washed with $3000 \mathrm{ml}$ of water and then eluted with $50 \%$ ethanol, concentrated under reduced pressure on a rotary evaporator, and then dried in a vacuum oven. A total of $1500 \mathrm{~g}$ of 200 300 mesh silica gel was suspended in $\mathrm{CHCl}_{3}$ and equilibrated the column with $\mathrm{CHCl}_{3}$ in a column. The sample was dissolved in $200 \mathrm{ml}$ of ethanol, mixed with 100 mesh silica gel, and dried at room temperature. After the column was equilibrated, the sample was loaded. $\mathrm{CHCl} 3: \mathrm{MeOH}=5: 1$ was used as the mobile phase to elute the sample successively. The eluted samples were collected ( $250 \mathrm{ml} /$ bottle), numbered, and analyzed via high-pressure liquid chromatography (HPLC) (apparatus: HPLC-SCL-10 Avp; mobile phase: $\mathrm{MeOH}: \mathrm{H} 2 \mathrm{O}$ (4:6); detection wavelength: $265 \mathrm{~nm}$; column size: Luna C18 $250 \times 4.6 \mathrm{~mm}$; column temperature: $40^{\circ} \mathrm{C}$; flow rate: $1 \mathrm{ml} / \mathrm{min}$; and injection volume: $10 \mu \mathrm{l})$. Thirty-nine grams of Saikosaponin was obtained. Finally, about $10 \mathrm{~g}$ of Saikosaponin A was finally purified on semipreparative HPLC (Beckman, Brea, CA, USA).

2.2. Establishment of the Model with SAP. Before the present study, all experimental processes were ensured to be consistent with the guidance for the care and use of laboratory animals from NIH and approved by the Animal Ethics Committee of The First Hospital of Jilin University (2017JLU0298). Eighty male Sprague-Dawley (SD) rats (8 weeks, 220-240 g) were purchased from the Animal Center of The First Hospital of Jilin University (Changchun, China). All rats were housed in separated cages (two rats in a cage) under either a 12:12 light:dark cycle (LD 12:12) and $22 \pm 1{ }^{\circ} \mathrm{C}$ with $65 \%$ humidity [2]. The rats had free access to standard food pellets and tap water ad libitum. SAP was established via the injection of $0.2 \mathrm{ml}$ of $5 \%$ sodium taurocholate into the biliopancreatic duct according to the previous report [31]. SAP was induced by sodium taurocholate that resulted in acinar cell calcium overload, zymogen activation, cytokine activities, and cell death [32]. The SAP model was confirmed by the evaluated levels of blood lipase or amylase [33]. Fifty microliters blood was obtained from each rat tail vein before and after 3-day model establishment, and serum was prepared via centrifugation at $2000 \times g$ for $10 \mathrm{~min}$. Rat serum amylase and lipase were measured by using the rat pancreatic amylase (PAMY) ELISA Kit (Cat. No. MBS269618) and rat pancreatic lipase ELISA Kit (Cat. No. MBS453575) from MyBioSource (San Diego, CA, USA) according to the manufacturer's instructions. For the controls, the rats were injected with $0.9 \% \mathrm{NaCl}$ solution. The animal model establishment was performed for twice as Figure 1 shows.

2.3. Animal Grouping. The dose range of Saikosaponin was referred to a report that the concentration of Saikosaponin A was from $6.25 \mathrm{mg} / \mathrm{kg}$ to $25.00 \mathrm{mg} / \mathrm{kg}$ [34], and a wider range of the dose $(10,20$, and $40 \mathrm{mg} / \mathrm{kg})$ was used in the present study. According to a previous report, pancreatic tissues 


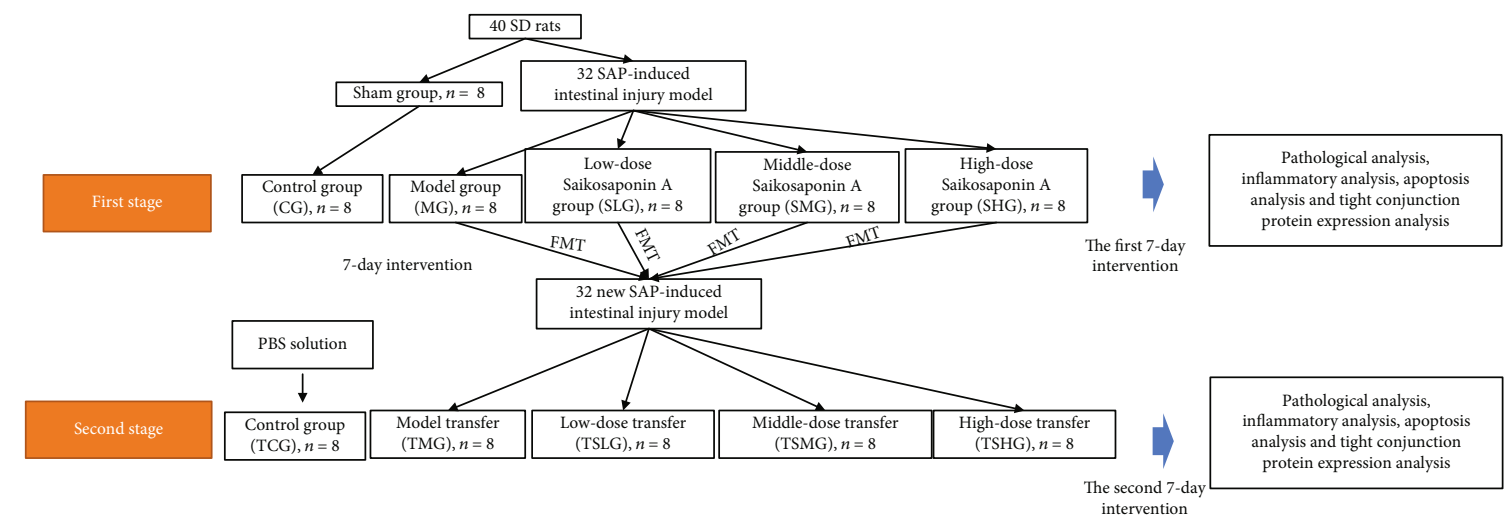

Figure 1: Study flow chart of the present experiment. There were 7 days for each stage, and the second stage was performed following the first stage.

were collected after 1-, 3-, and 5-day SAP model establishment. Most inflammatory cytokines had significant changes after 3- and 5-day SAP model establishment [35]. On the other hand, considering the effects of the Saikosaponin A on gut microbiota, a longer time $(7 \mathrm{~d})$ may be used [36]. There were two stages for the whole experiment as Figure 1 shows. After 12-hour sodium taurocholate injection, 24 SAP rats were administrated with different concentrations of Saikosaponin A $(10,20$, and $40 \mathrm{mg} / \mathrm{kg})$ in $0.2 \mathrm{ml} 0.9 \%$ saline solution via the tail vein. Meanwhile, $0.2 \mathrm{ml} 0.9 \%$ saline solution was injected in the control or animal group $(n=8$ for each group) without Saikosaponin A. In the first stage, all rats were divided into CG (control group), MG (model group), SLG (low-dose Saikosaponin A), SMG (middle-dose Saikosaponin A), and SHG (high-dose Saikosaponin) groups according to different treatments (Figure 1). Rats were monitored daily for weight and disease activity index (DAI, including weight loss, presence of blood in feces, and stool consistency). The feces and urine samples were collected at 7 days. After 7 days, the rats were anesthetized with pentobarbital $(40 \mathrm{mg} / \mathrm{kg}$ i.p.), blood samples were drawn, colon length was measured, and the animals were killed. Pancreas tissues were quickly removed and frozen at $-80^{\circ} \mathrm{C}$ until use.

After 7-day Saikosaponin A intervention, fecal sample $(1.0 \mathrm{~g})$ was obtained from each rat in the MG, SLG, SMG, and SHG groups, suspended with $5 \mathrm{ml}$ of sterile PBS buffer, and used to colonize the newly SAP rats. In the second stage, fecal microbiota transplantation (FMT) was transferred according to the previous study [37]. For the control group, the rats were treated with PBS buffer. Briefly, the fecal samples in the PBS solution (0.01 M, pH 7.4) were vortexed for $5 \mathrm{~min}$, homogenized, and centrifuged for $10 \mathrm{~min}(1,000 \mathrm{~g})$, and the pools were divided into equal volumes for $7 \mathrm{~d}$. The model rats were colonized with pooled samples for another $7 \mathrm{~d}$. These rats were divided into TCG, TMG, TSLG, TSMG, and TSHG groups according to the fecal microbiota were from MG, SLG, SMG, and SHG groups, respectively (Figure 1).

2.4. DAI. The DAI was evaluated according to the previous report, and the DAI score was presented as mean scores of body weight loss, fecal consistency, and fecal blood test scores [38].

2.5. Histological Evaluation. Colon tissues were washed in PBS buffer. The pancreas tissues were fixed by using $4 \%$ formalin, embedded in paraffin, cut in $4 \mathrm{~m}$ sections, and stained with hematoxylin and eosin (H\&E) [39]. The inflammatory and injury of pancreas were observed in 5 fields per sections. The pathological grades were calculated according to Park's classification $[40,41]$ with slight modification: Grade 0 , the lowest grade of injury with normal pancreas cell structure and without inflammation; Grade 1, the normal pancreas morphology was with a small number of mononuclear cell infiltration; Grade 2, the pancreas tissues were with middle number of mononuclear cell infiltration; Grade 3, the pancreas tissues were characterized with high-number of mononuclear cell infiltration; and Grade 4, pancreas structure was damaged with the significant number of mononuclear cell infiltration.

2.6. Serum Oxidative Assay. Blood sample was collected via abdominal aorta and centrifuged at $4000 \times g$ for $10 \mathrm{~min}$ at $4^{\circ} \mathrm{C}$. The serum was separated and stored at $-80^{\circ} \mathrm{C}$ for subsequent biochemical testing. Sera MDA, CAT, SOD, and GPx were measured by using corresponding assay kits from Northwest Life Science Specialties (Vancouver, WA, USA), Cayman Chemical Company (MI, USA), Calbiochem (San Diego, CA, USA), and Nanjing Jiancheng Bio-Tek Co. (Nanjing, China) on an automatic blood chemical analyzer (CIBA Corning, OH, USA), respectively.

2.7. Serum Inflammatory Cytokines Analysis. The levels of TNF- $\alpha$ (SKU: BC-ER141303), IL-1 $\beta$ (SKU: BC-EH101933), IL-6 (SKU: BC-ER140741), and IL-10 (SKU: BC-ER140711) in serum were assessed by using ELISA kits following the manufacturer's scheme from Biocodon Technologies (Mission, KS, USA) and an automatic blood chemical analyzer (CIBA Corning, OH, USA). Serum C-reactive protein (CRP) was measured using the ELISA kit from DRG Instrument $\mathrm{GmbH}$ (Marburg, Germany) on a microplate reader from Thermo Scientific (Waltham, MA, USA). 
Serum procalcitonin (PCT) was measured with the ELISA kit from EIAab Science Co., Ltd. (Wuhan, China).

2.8. Reverse Transcription-Quantitative PCR (RT-qPCR). RNA was isolated from $5 \mathrm{mg}$ pancreas using TRIzol reagent (Shanghai Shenggong Co., Ltd., Shanghai, China). cDNA was made by using a reverse transcription kit (Shanghai Shenggong Co., Ltd., Shanghai, China). The following primers were used: Keap1 forward primer $5^{\prime}$-TTCGCCT ACACGGCCTC- $3^{\prime}$ and reverse primer $5^{\prime}$-GAAGTTGGC GATGCCGATG-3'; Nrf2, forward primer $5^{\prime}$-CCTCAACT ATAGCGATGCTGAATCT- $3^{\prime}$ and reverse primer $5^{\prime}$ AGGAGTTGGGCATGAGTGAGTAG-3'; ARE, forward primer $5^{\prime}$-CTGTCCTCAAATGAACCTGCCTCCTC- $3^{\prime}$ and reverse primer $5^{\prime}$-GAGGAGGCAGGTTCCATTGAGGAC AG- $3^{\prime}$; and $\beta$-actin, forward primer $5^{\prime}$-AAGTCCCTCAC CCTCCCAAAAG- $3^{\prime}$ and reverse primer $5^{\prime}$-AAGCAATGC TGTCACCTTCCC-3'. RT-qPCR was performed on GeneAmp PCR System 9700 (Applied Biosystems, Foster City, CA, USA). The levels of target genes were detected and the relative mRNA levels were normalized to $\beta$-actin using the $2^{-\Delta \Delta \mathrm{Ct}}$ method.

2.9. Western Blot. Pancreas tissues were used to measure the expression of Keap1, Nrf2, and ARE. Ten milligrams of pancreatic tissue was ground in liquid nitrogen, and total protein was extracted by using the Protein Isolation Kit (Invitrogen, CA, USA) according to the manufacture's instruction. Protein concentration was determined by using the BCA kit (Invitrogen, Carlsbad, CA, USA). HRP-conjugated goat anti-rabbit IgG H\&L (ab6721) secondary antibodies were from Abcam (Abcam, San Francisco, CA, USA). The proteins were separated by SDS-PAGE and transferred to the PVDF membrane. The membrane was blocked for 1 hour at ambient room temperature in $10 \%$ nonfat milk and incubated with primary antibody (Anti-Keap1 antibody/AntiNrf2 antibody/Anti-ARE antibody from Abcam (1:1,000; Cambridge, MA, USA) overnight at $4^{\circ} \mathrm{C}$. The membrane was rinsed 3 times with PBTB, incubated 2 hours at $37^{\circ} \mathrm{C}$ in secondary antibodies. Image was obtained on an infrared scanner (Odyssey, Lincoln, NE, USA). Relative protein levels were calculated by using internal reference $\beta$-actin.

2.10. Analysis of Gut Microbiota. Fecal pellets were collected and weighed, homogenized with $1 \mathrm{ml}$ of sterile PBS. Bacterial DNA was extracted from the samples by using the QIAamp Fast DNA Stool Mini Kit (catalog number: 51604, QIAGEN, CA, USA). The isolated DNA was amplified using primers for the target gene 16S rRNA (V3-4 regions: forward $5^{\prime}$ CCTACGGGNGGCWGCAG- $3^{\prime}$ and reverse $5^{\prime}$-GACTAC HVGGGTATCTAATCC- $3^{\prime}$ ) according to the previous report [42]. The gut microbiota of rats was analyzed by using $16 \mathrm{~S}$ rRNA sequencing of bacterial genomes.

2.11. Statistical Analysis. All data were presented as the mean \pm standard deviation (S.D.). The variables were analyzed by using unpaired two tailed Student's $t$-test. A normal distribution of variance was confirmed by the Kolmogorov and Smirnov test [43]. Homogeneity of variance was confirmed by using Bartlett's test [44]. $p$ values were corrected for multiple comparisons by using the Bonferroni adjustment [45]. The statistical data were analyzed by one-way ANOVA followed by Tukey's post hoc test. The statistical difference was considered if $p<0.5$.

\section{Results}

3.1. HPLC Analysis of Saikosaponin A. Comparing with the standard of Saikosaponin A (Figure 2(a)), HPLC analysis showed that the main extracts of Radix bupleuri was Saikosaponin A (Figure 2(b)) and the eluting time was $19.9 \mathrm{~min}$. Radix bupleuri may exert its function via its main component Saikosaponin A.

3.2. Saikosaponin A Intervention Reduced SAP Symptoms. The present results showed that the levels of serum amylase (Figure 3(a)) and serum lipase (Figure 3(b)) significantly increased in the MG group when compared with the CG group $(p<0.05)$. The results suggested that SAP was established with the significantly increased levels of serum amylase and lipase. On the other hand, Saikosaponin A treatment reduced the level of serum amylase (Figure 3(a)) and serum lipase (Figure $3(\mathrm{~b}))$ in a dose-dependent way $(p<0.05)$. The results suggest that Saikosaponin A intervention reduces serum levels of amylase and lipase in the SAP model.

To explore the effects of Saikosaponin A on pancreas damage, an SAP model was established in rats. The DAI was 0 in the CG group, and the scores were highest in the MG group. Saikosaponin A intervention reduced the DAI value in a dose-dependent way (Figure $4(a), p<0.05$ ). Pathological change was assessed by using the H\&E stain. There was no pathological character in the CG group with Grade 0 . In contrast, the rats had a significant pathological character in the MG group with grade 4, including acinar cell edema, widened intercellular spaces, hemorrhage, necrosis, inflammatory cell infiltration, and cell destruction (Figures 4(b) and $3(c), p<0.05)$. The pathological characters were significantly reduced in Saikosaponin A-treated group in a dosedependent way (Figures 4(b) and 3(c), $p<0.05$ ). These results suggest that Saikosaponin A intervention ameliorates SAP lesions.

3.3. Saikosaponin A Had Antioxidant and AntiInflammatory Effects on the Rats with SAP. Antioxidant analysis showed that serum levels of SOD (Figure 5(a)), CAT (Figure 5(b)), and GPx (Figure 5(c)) were highest in the $C G$ group and significantly reduced in the $M G$ group while the MDA level was lowest in the CG group and highest in the MG group (Figure 5(d), $p<0.05$ ). Saikosaponin A intervention increased the serum levels of SOD (Figure 5(a)), CAT (Figure 5(b)), and GPx (Figure 5(c)) and reduced the MDA level (Figure 5(d), $p<0.05$ ). The administration of Saikosaponin A suppressed oxidative stress in the rats with SAP.

Anti-inflammatory analysis showed that serum levels of TNF- $\alpha$ (Figure 5(e)), IL-1 $\beta$ (Figure 5(f)), and IL-6 (Figure $5(\mathrm{~g})$ ) were lowest in the CG group and significantly 


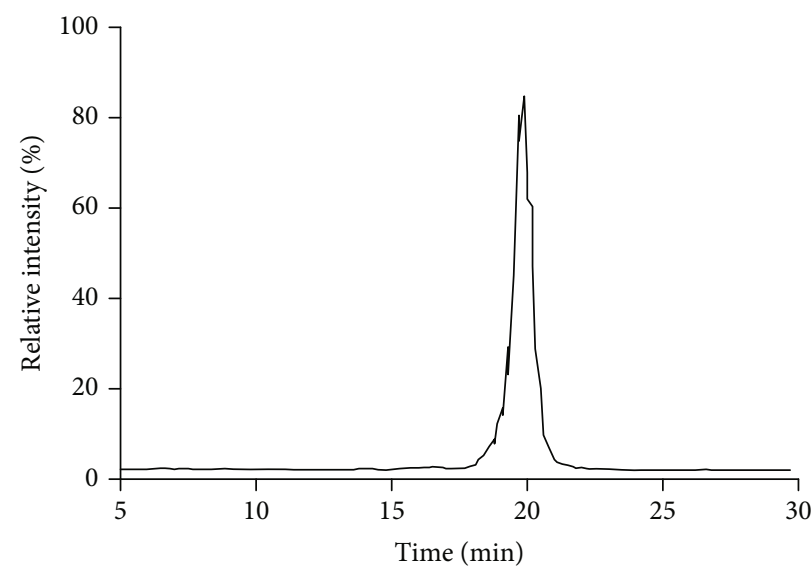

(a)

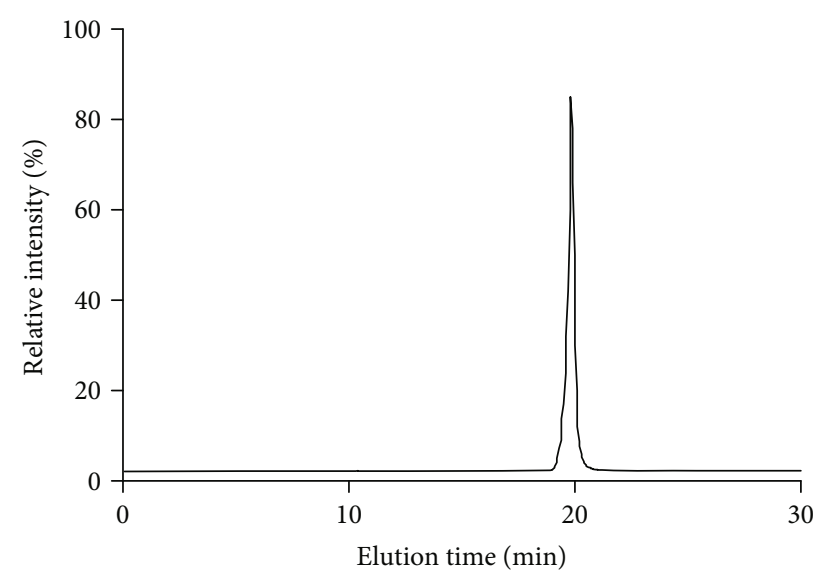

(b)

FIGURE 2: High-performance liquid chromatography (HPLC) analysis for the extracts of Radix bupleuri. (a) The standard of Saikosaponin A. (b) Purified Saikosaponin A.

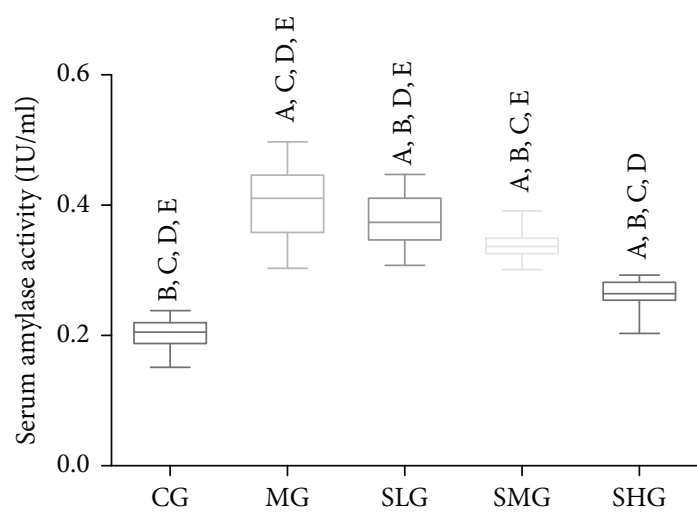

(a)

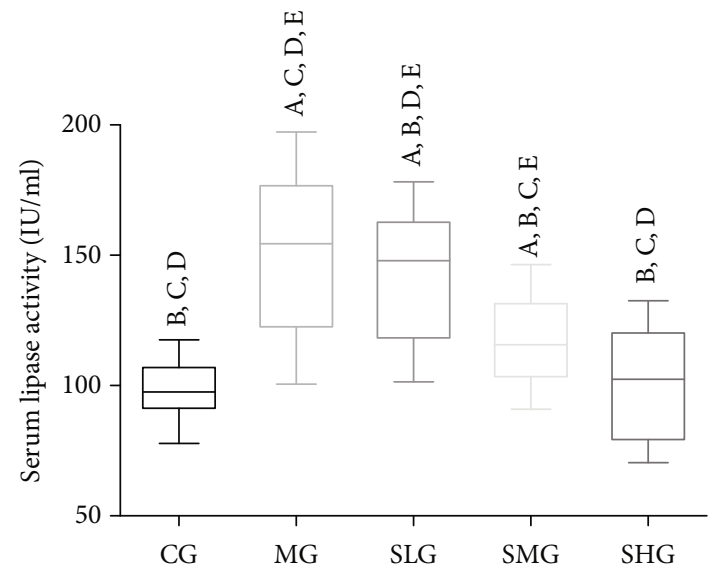

(b)

Figure 3: The effects of Saikosaponin A on serum lipase and amylase among different groups. Data were presented as means \pm S.D. (standard deviation) and $n=8$ for each group. ${ }^{\mathrm{a}} p<0.05$ vs. the CG group, ${ }^{\mathrm{b}} p<0.05$ vs. the MG group, ${ }^{\mathrm{c}} p<0.05$ vs. the SLG group, ${ }^{\mathrm{d}} p<0.05$ vs. the SMG group, and ${ }^{\mathrm{e}} p<0.05$ vs. the SHG group.

increased in the MG group while the IL-10 level was highest in the CG group and lowest in the MG group (Figure 5(h), $p<0.05)$. Saikosaponin A intervention reduced the serum levels of TNF- $\alpha$ (Figure 5(e)), IL-1 $\beta$ (Figure 5(f)), and IL-6 (Figure 5(g)) and increased the IL-10 level (Figure 5(h), $p<$ 0.05 ). The levels of serum CRP (Figure 5(i)) and serum PCT (Figure 5(j)) significantly increased in the MG group when compared with the CG group $(p<0.05)$. The results suggested that SAP was established with the significantly increased levels of serum CRP and PCT. On the other hand, Saikosaponin A treatment reduced the level of serum CRP (Figure 5(i)) and serum PCT (Figure 5(j)) in a dosedependent way $(p<0.05)$. The results suggest that Saikosaponin A intervention reduces serum levels of inflammatory indicators in the SAP model. Saikosaponin A increased anti-inflammatory properties in the rats with SAP.

3.4. Saikosaponin A Increased the Relative mRNA Levels of Antioxidant Signaling Molecules. Keap1-Nrf2-ARE are impor- tant antioxidant signaling molecules to maintain antioxidant properties and pancreas integrity [46]. Thus, we explored the protective function by investigating the effects of Saikosaponin A on the relative mRNA levels of the antioxidant signaling. The levels of Keap1 (Figure 6(a)), Nrf2 (Figure 6(b)), and ARE (Figure 6(c)) were obviously decreased in the MG group when compared with those in the CG group $(p<0.05)$. However, the administration of Saikosaponin A increased the level of Keap1 (Figure 6(a)), Nrf2 (Figure 6(b)), and ARE (Figure 6(c)). The experiment result demonstrated that Saikosaponin A ameliorated the SAP by increasing the relative mRNA levels of antioxidant signaling molecules.

3.5. Saikosaponin A Increased the Protein Levels of Antioxidant Signaling Molecules. We further explored the protective function by investigating the effects of Saikosaponin A on the protein levels of the antioxidant signaling molecules. The expression levels of Keap1 (Figure 7(a)), Nrf2 (Figure 7(b)), and ARE (Figure 7(c)) obviously decreased in 


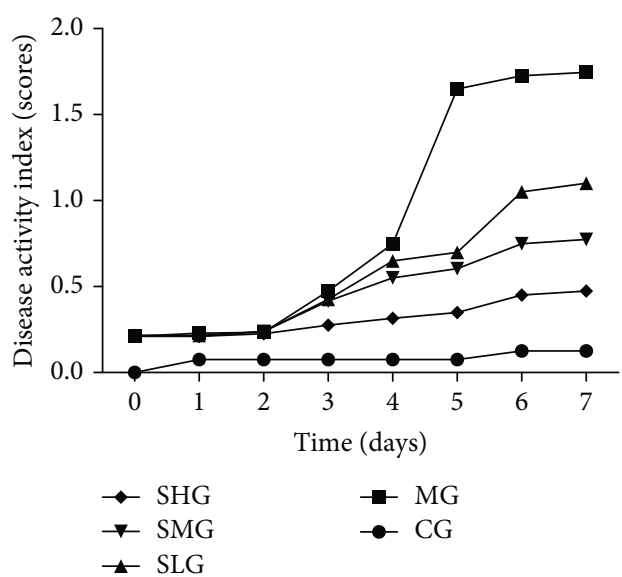

(a)

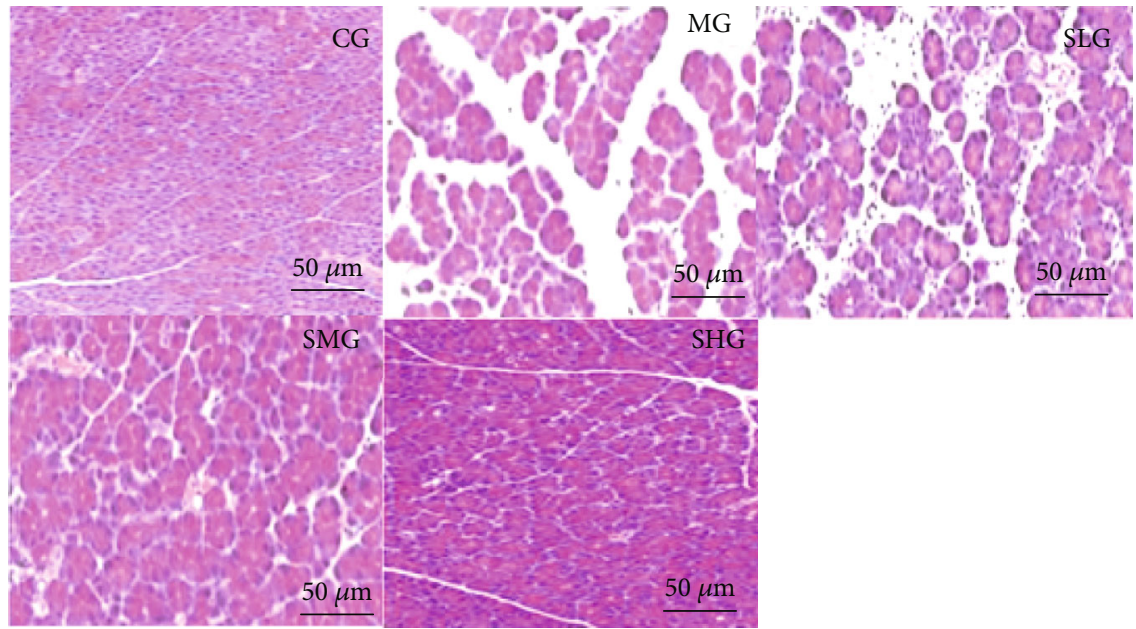

(b)

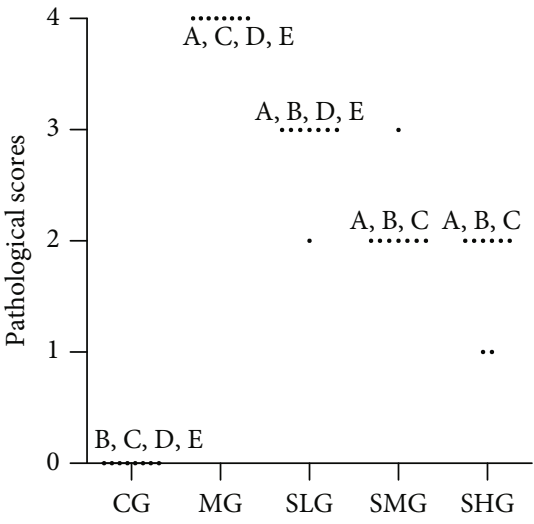

(c)

FIgure 4: Saikosaponin A ameliorated pathological character of SAP in rats. (a) Disease activity index. (b) Hematoxylin and eosin (H\&E) staining of pancreas in each group. (c) Histopathological scores. Data were presented as means \pm S.D. (standard deviation) and $n=8$ for each group. ${ }^{\mathrm{a}} p<0.05$ vs. the CG group, ${ }^{\mathrm{b}} p<0.05$ vs. the MG group, ${ }^{\mathrm{c}} p<0.05$ vs. the SLG group, ${ }^{\mathrm{d}} p<0.05$ vs. the SMG group, and $\mathrm{e}^{\mathrm{e}}<<0.05$ vs. the SHG group. 


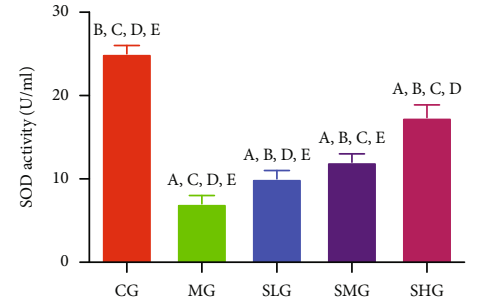

(a)

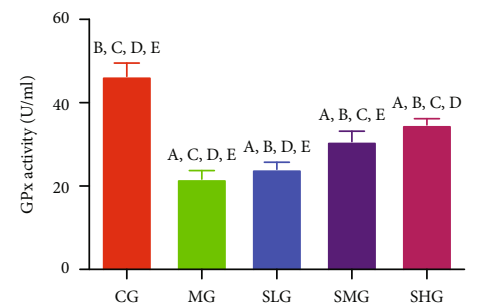

(c)

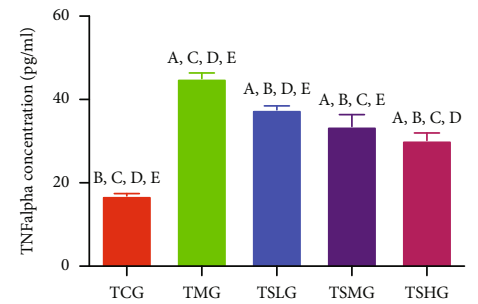

(e)

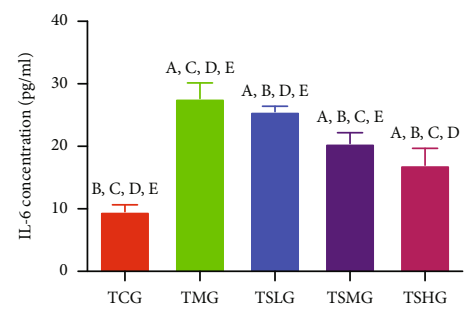

(g)

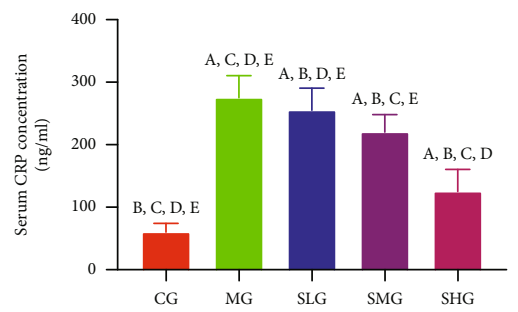

(i)

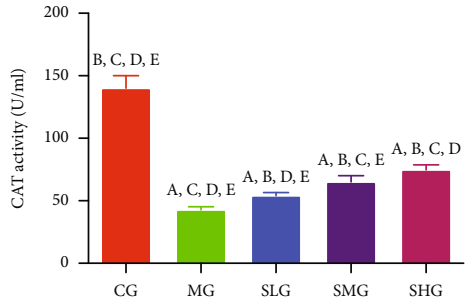

(b)

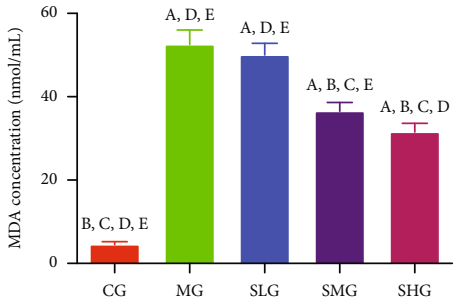

(d)

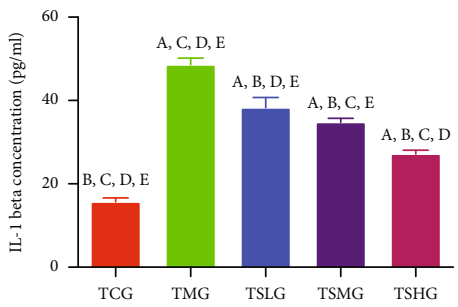

(f)

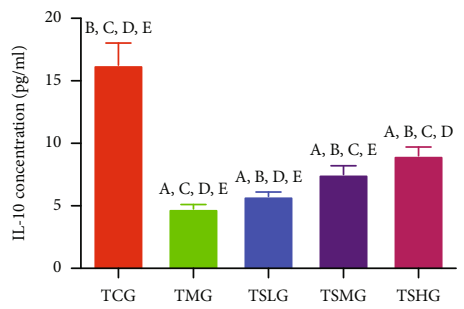

(h)

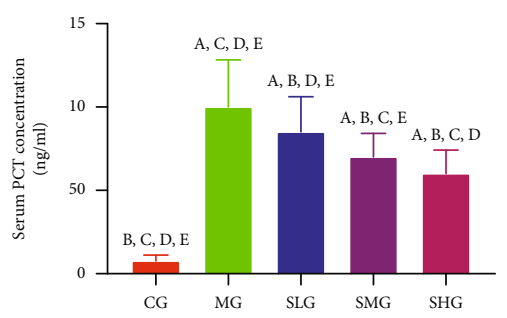

(j)

FIgURE 5: The effects of administration of Saikosaponin A on serum level of oxidative stress, inflammatory responses, and apoptosis status in the rats with severe acute pancreatitis (SAP). (a) Superoxide dismutase (SOD). (b) Catalase (CAT). (c) Oxidized glutathione (GPx). (d) Malondialdehyde (MDA). (e) Tumor necrosis factor- (TNF-) $\alpha$. (f) Interleukin- (IL-) 1 $\beta$. (g) IL-6. (h) IL-10. (i) C-reactive protein (CRP). (j) Procalcitonin (PCT). Data were presented as means \pm S.D. (standard deviation) and $n=8$ for each group. ${ }^{a} p<0.05$ vs. the CG group, ${ }^{\mathrm{b}} p<0.05$ vs. the MG group, ${ }^{\mathrm{c}} p<0.05$ vs. the SLG group, ${ }^{\mathrm{d}} p<0.05$ vs. the SMG group, and ${ }^{\mathrm{e}} p<0.05$ vs. the SHG group.

the MG group when compared with those in the CG group $(p<0.05)$. However, the administration of Saikosaponin A increased the expression levels of Keap1 (Figure 7(a)), Nrf2 (Figure 7(b)), and ARE (Figure 7(c)). The experiment result demonstrated that Saikosaponin A ameliorated the SAP status by increasing the expression of antioxidant signaling proteins.

3.6. Saikosaponin A Intervention Improved Gut Microbiota Composition. Dysbiosis of gut microbiota is closely 


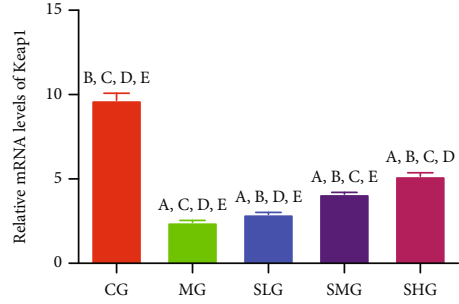

(a)

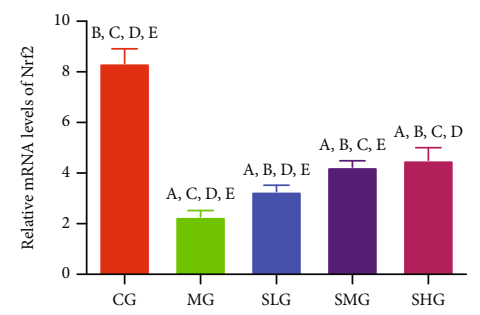

(b)

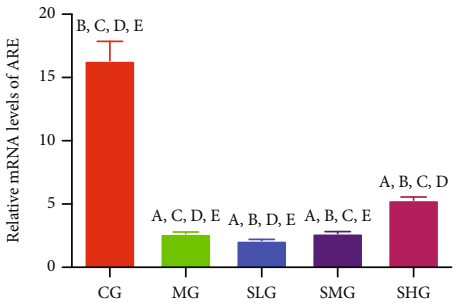

(c)

Figure 6: The real-time reverse transcription polymerase chain reaction (RT-PCR) analysis of the effects of Saikosaponin A on the relative mRNA levels of antioxidant signaling proteins. (a) Kelch-like ECH-associated protein 1 (Keap1). (b) Nuclear factor erythroid 2-related factor 2 (NRF2). (c) Antioxidant response element (ARE). Data were presented as mean values \pm S.D. (standard deviation) and $n=8$ for each group. ${ }^{\mathrm{a}} p<0.05$ vs. the CG group, ${ }^{\mathrm{b}} p<0.05$ vs. the MG group, ${ }^{\mathrm{c}} p<0.05$ vs. the SLG group, ${ }^{\mathrm{d}} p<0.05$ vs. the SMG group, and $\mathrm{e}_{p}<0.05$ vs. the SHG group.

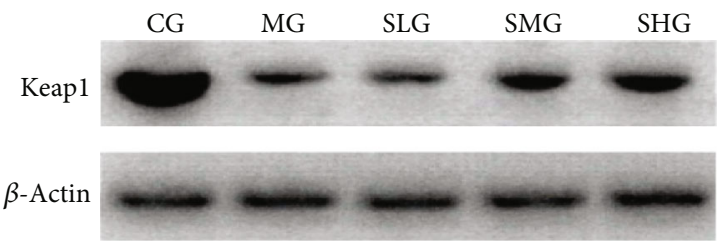

(a)

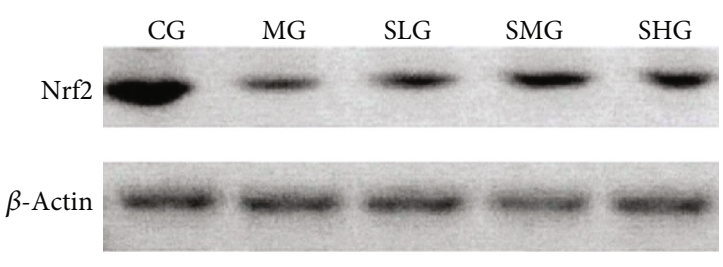

(b)
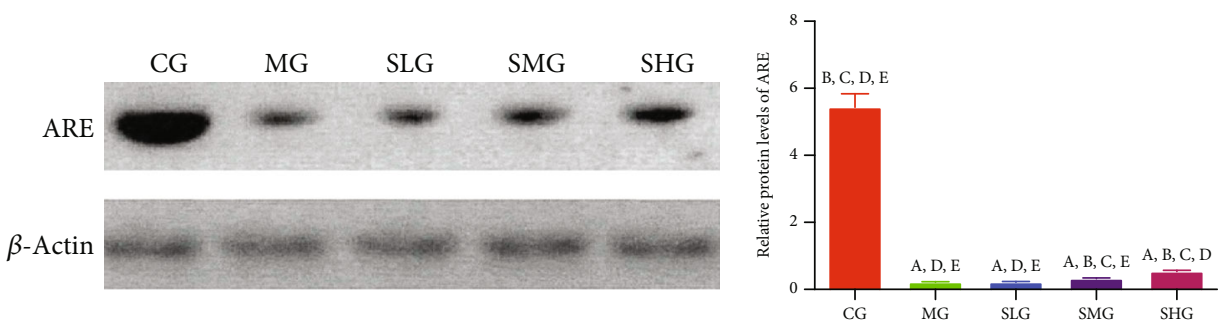

(c)

FIgURE 7: Western blot analysis of the effects of Saikosaponin A on the expression of antioxidant signaling proteins. (a) Kelch-like ECHassociated protein 1 (Keap1). (b) Nuclear factor erythroid 2-related factor 2 (NRF2). (c) Antioxidant response element (ARE). Data were presented as mean values \pm S.D. (standard deviation) and $n=8$ for each group. ${ }^{\mathrm{a}} p<0.05$ vs. the CG group, ${ }^{\mathrm{b}} p<0.05$ vs. the MG group, ${ }^{c} p<0.05$ vs. the SLG group, ${ }^{\mathrm{d}} p<0.05$ vs. the SMG group, and ${ }^{\mathrm{e}} p<0.05$ vs. the SHG group.

associated with pancreas dysfunction and damage. To investigate the impact of Saikosaponin A on gut microbiota composition, 16S rRNA gene sequencing was conducted by using the different fecal specimens from all groups. The relative abundance of Lactobacillus species was highest in the CG, and lowest in the MG group (Figure 8(a)). Saikosaponin A treatment increased the relative abundance of Lactobacillus species in a dose-dependent way (Figure 8(a)). The abun- dance of Prevotella species was similar between the CG and MG groups (Figure 8(a)). Saikosaponin A treatment increased the relative abundance of Prevotella species in a dose-dependent way (Figure 8(a)). Heatmap analysis showed the similar results as those in the barplots (Figure 8(b)).

3.7. FMT of Saikosaponin A-Treated Rats Ameliorated SAP Lesions. To explore whether the gut microbiota of 


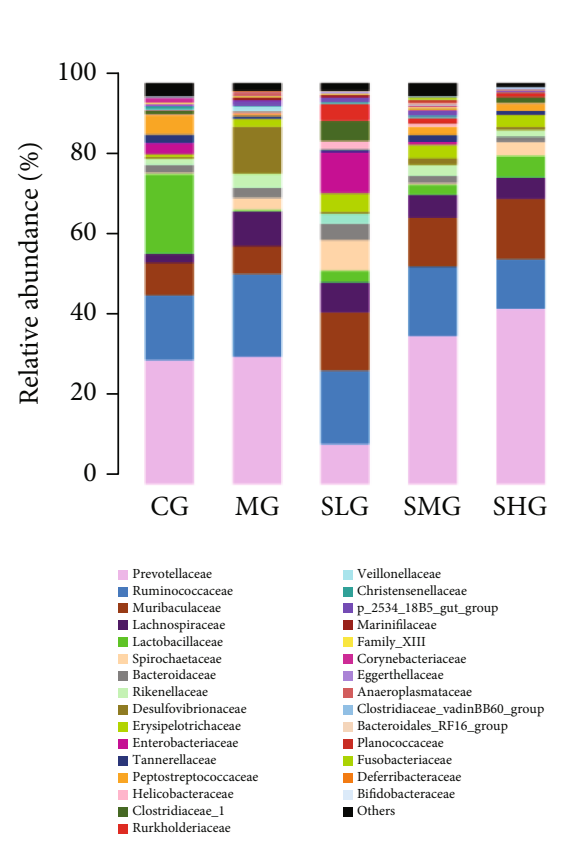

(a)

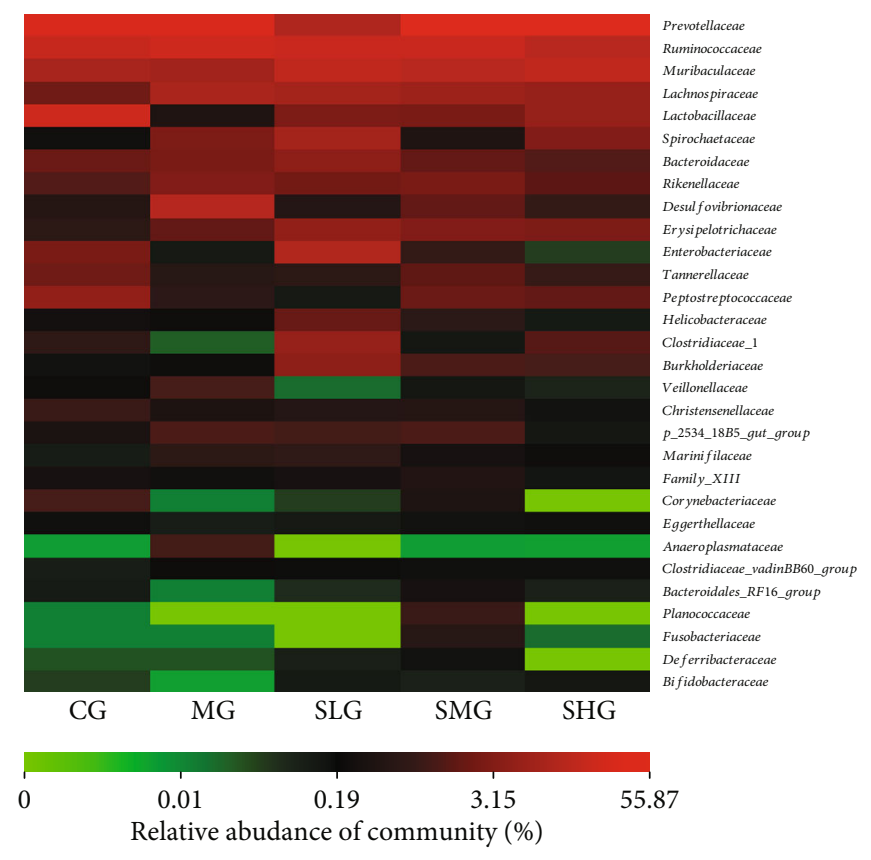

(b)

Figure 8: The composition of gut microbiota among different groups. (a) The proportion of gut microbiota. (b) Heatmap analysis of gut microbiota changes from different treatments.

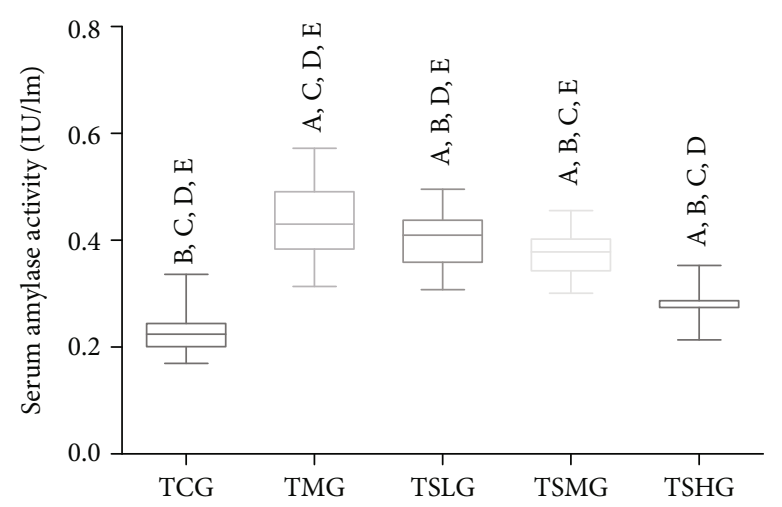

(a)

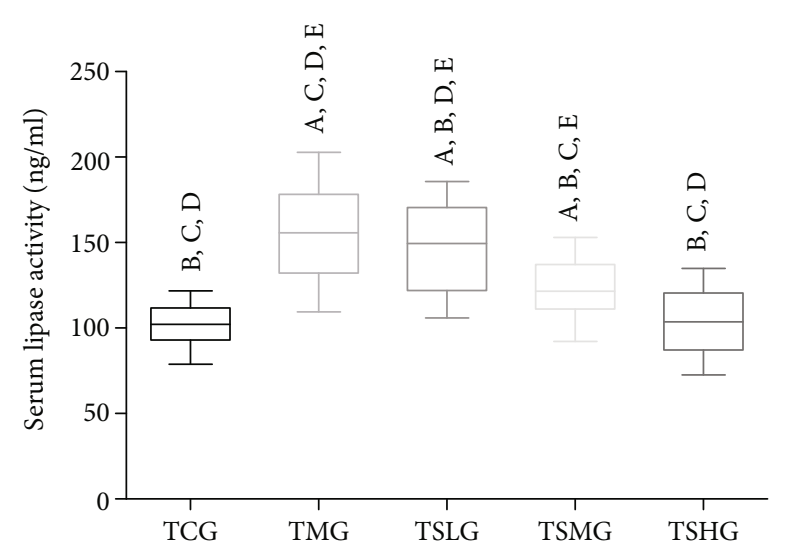

(b)

FIGURE 9: The effects of fecal microbiota transplantation (FMT) of Saikosaponin A-treated rats on serum lipase and amylase among different groups. Data were presented as means \pm S.D. (standard deviation) and $n=8$ for each group. ${ }^{\mathrm{a}} p<0.05$ vs. the TCG group, ${ }^{\mathrm{b}} p<0.05$ vs. the TMG group, ${ }^{c} p<0.05$ vs. the TSLG group, ${ }^{\mathrm{d}} p<0.05$ vs. the TSMG group, and ${ }^{\mathrm{e}} p<0.05$ vs. the TSHG group.

Saikosaponin A-treated animals improve the SAP rats, the gut microbiota of Saikosaponin A-treated SAP rats (the model was established within the first 7 days) was transferred to another SAP rats (the model was established within the second 7 days). All the previous parameters were repeated analyzed. The levels of serum amylase (Figure 9(a)) and serum lipase (Figure 9(b)) significantly increased in the TMG group when compared with the TCG group $(p<0.05)$. The results suggested that FMT of the SAP model rats significantly increased the levels of serum amylase and lipase. On the other hand, FMT of Saikosaponin A-treated rats reduced the level of serum amylase (Figure 9(a)) and serum lipase (Figure 9(b), $p<0.05)$. The results suggest that FMT of Saikosaponin A-treated rats reduces serum levels of amylase and lipase in the SAP model. FMT of model rat increased disease activity index scores (Figure 10(a)) and pathological scores (Figures 10(b) and 10(c)). In contrast, the FMT of Saikosaponin A-treated rats reduced disease activity index scores (Figure 10(a)) and pathological scores (Figures 10(b) and 10(c)). These results suggest that Saikosaponin A ameliorates SAP lesions by improving gut microbiota.

3.8. FMT of Saikosaponin A-Treated Rats Had Antioxidant and Anti-Inflammatory Effects on the Rats with SAP. Antioxidant analysis showed that serum levels of SOD 


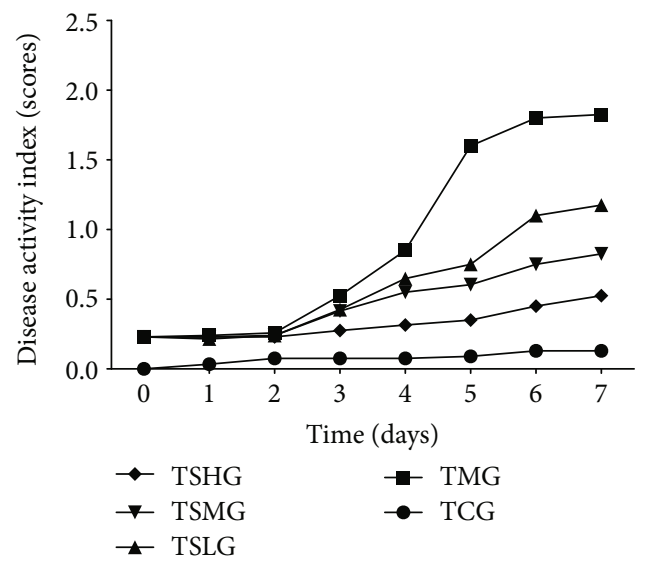

(a)

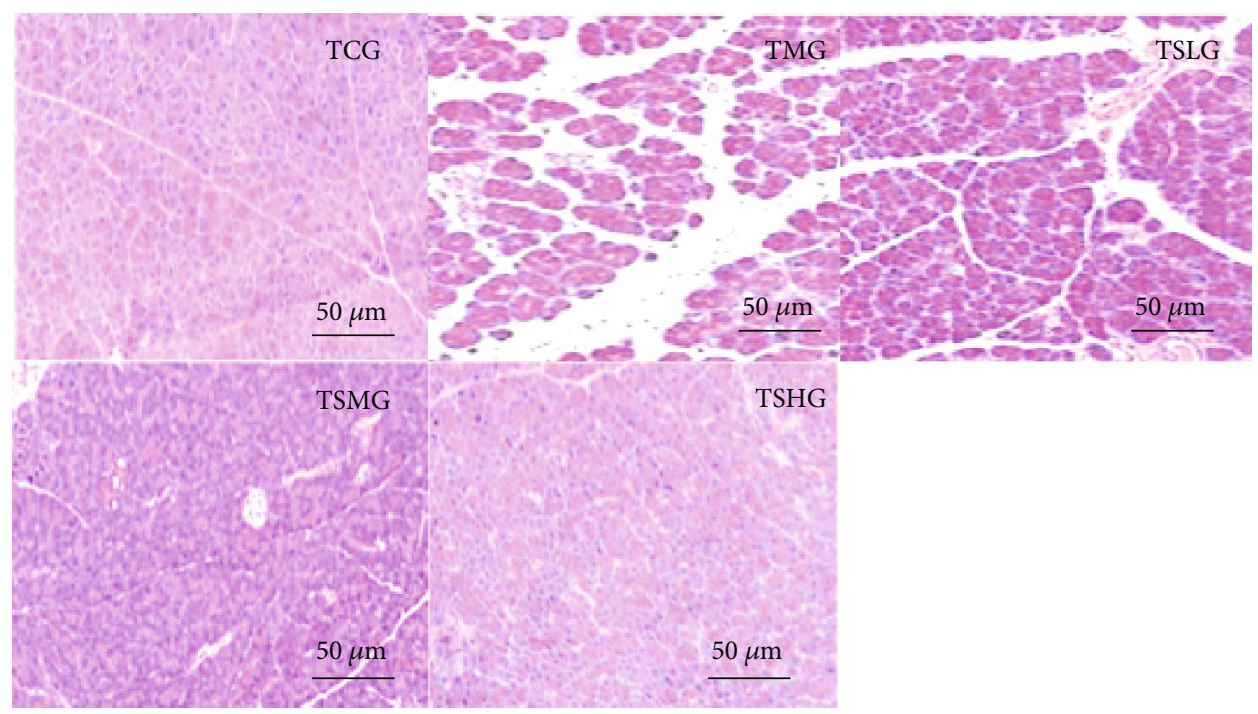

(b)

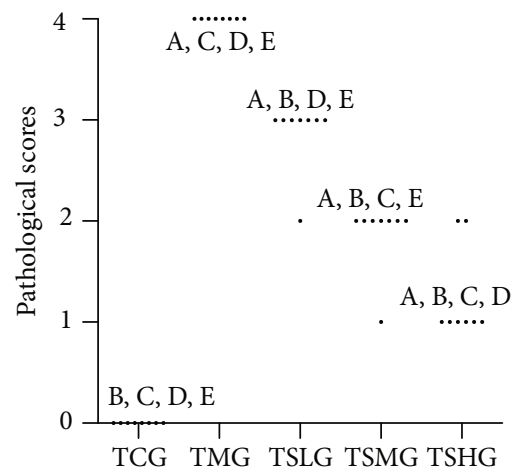

(c)

FIGURE 10: Fecal microbiota transplantation (FMT) of Saikosaponin A-treated rats ameliorated pathological character of severe acute pancreatitis (SAP) in rats. (a) Disease activity index. (b) Hematoxylin and eosin (H\&E) staining of pancreas in each group. (c) Histopathological scores. Data were presented as means \pm S.D. (standard deviation) and $n=8$ for each group. ${ }^{a} p<0.05$ vs. the TCG group, ${ }^{\mathrm{b}} p<0.05$ vs. the TMG group, ${ }^{\mathrm{c}} p<0.05$ vs. the TSLG group, ${ }^{\mathrm{d}} p<0.05$ vs. the TSMG group, and $\mathrm{e}^{\mathrm{T}}<0.05$ vs. the TSHG group.

(Figure 11(a)), CAT (Figure 11(b)), and GPx (Figure 11(c)) were highest in the TCG group and significantly reduced in the TMG group while the MDA level was lowest in the
TCG group and highest in the TMG group (Figure 11(d), $p<0.05)$. FMT of Saikosaponin A-treated rats increased the serum levels of SOD (Figure 11(a)), CAT (Figure 11(b)), 


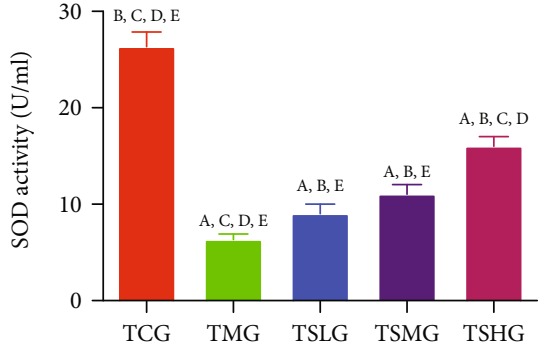

(a)

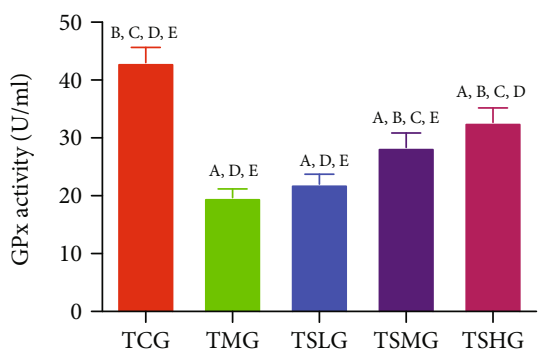

(c)

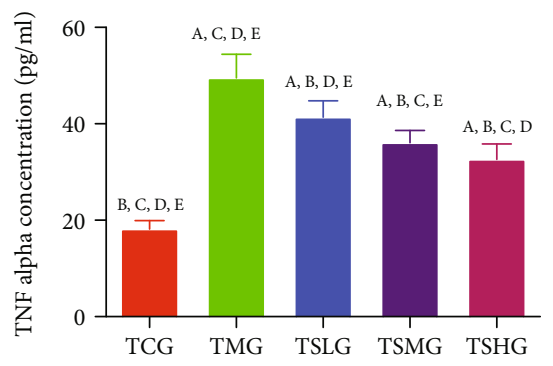

(e)

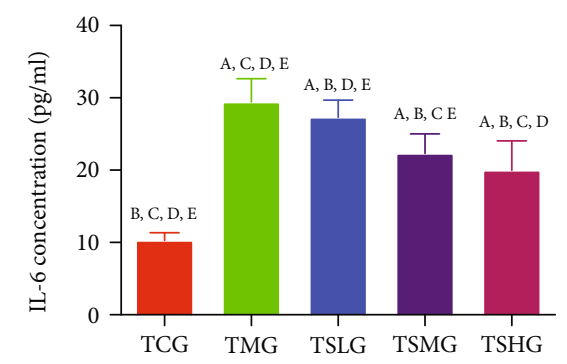

(g)

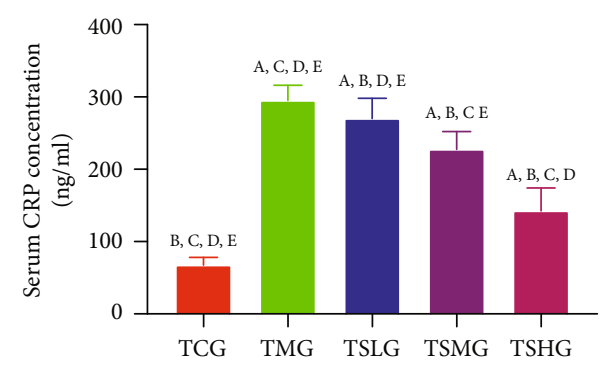

(i)

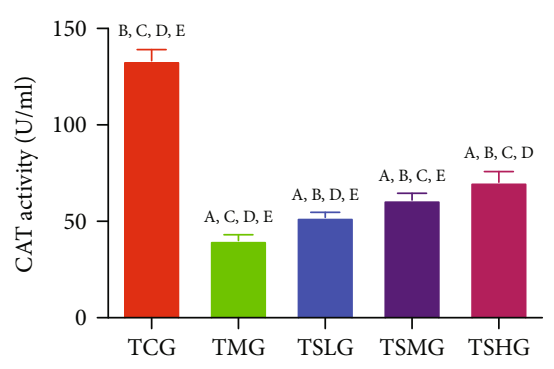

(b)

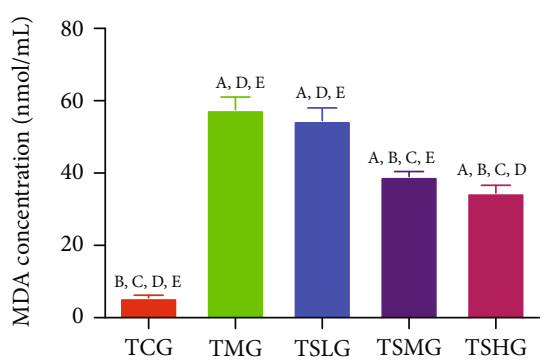

(d)

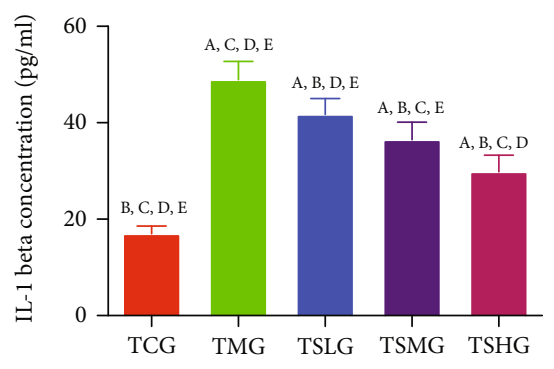

(f)

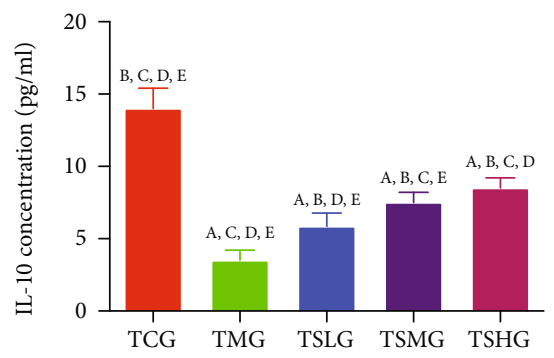

(h)

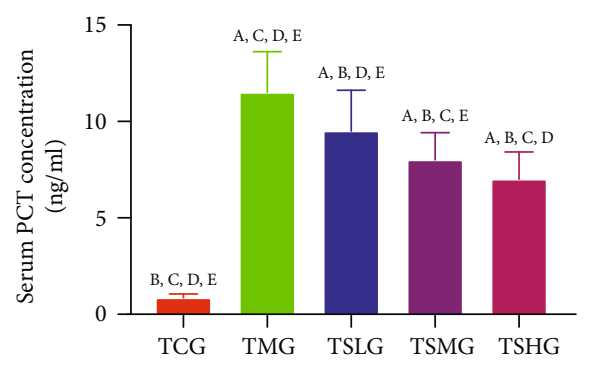

(j)

Figure 11: The effects of Saikosaponin A-treated rats on serum level of oxidative stress, inflammatory responses, and apoptosis status in the rats with severe acute pancreatitis (SAP). (a) Superoxide dismutase (SOD). (b) Catalase (CAT). (c) Oxidized glutathione (GPx). (d) Malondialdehyde (MDA). (e) Tumor necrosis factor- (TNF-) $\alpha$. (f) Interleukin- (IL-) 1 $\beta$. (g) IL-6. (h) IL-10. (i) C-reactive protein (CRP). (j) Procalcitonin (PCT). Data were presented as means \pm S.D. (standard deviation) and $n=8$ for each group. ${ }^{a} p<0.05$ vs. the TCG group, ${ }^{\mathrm{b}} p<0.05$ vs. the TMG group, ${ }^{\mathrm{c}} p<0.05$ vs. the TSLG group, ${ }^{\mathrm{d}} p<0.05$ vs. the TSMG group, and ${ }^{\mathrm{e}} p<0.05$ vs. the TSHG group. 


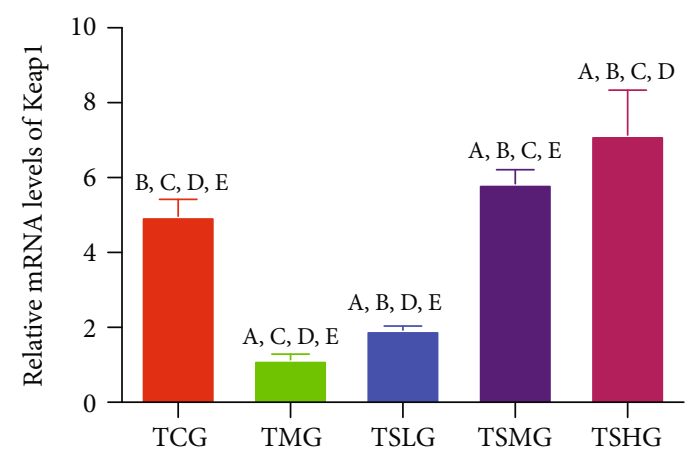

(a)

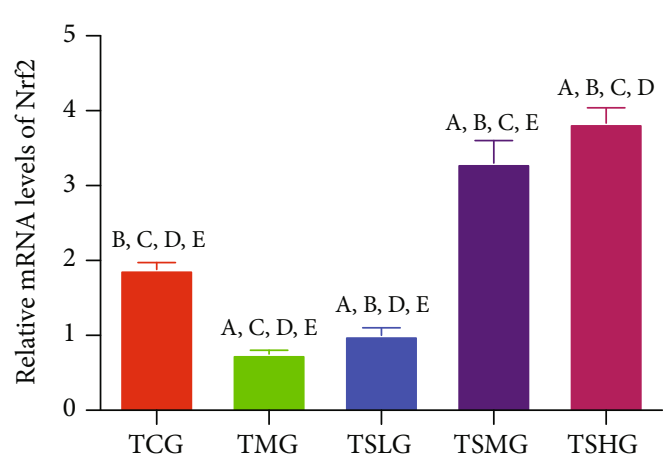

(b)

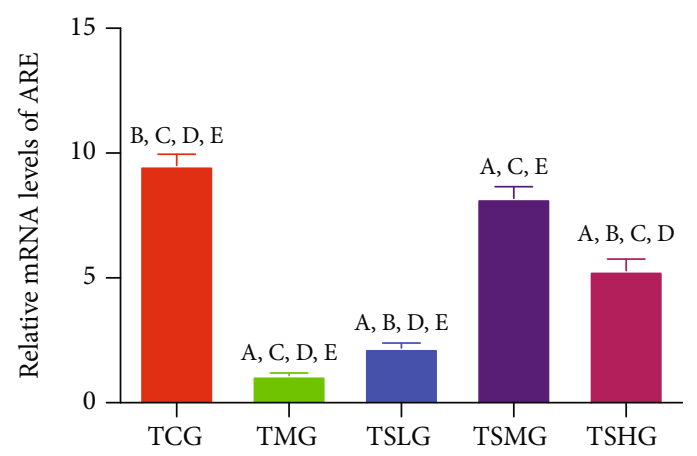

(c)

FIGURE 12: The real-time reverse transcription polymerase chain reaction (RT-PCR) analysis of the effects of fecal microbiota transplantation (FMT) of Saikosaponin A on relative mRNA levels of antioxidant signaling proteins. (a) Kelch-like ECH-associated protein 1 (Keap1). (b) Nuclear factor erythroid 2-related factor 2 (NRF2). (c) Antioxidant response element (ARE). Data were presented as means \pm S.D. (standard deviation) and $n=8$ for each group. ${ }^{\mathrm{a}} p<0.05$ vs. the TCG group, ${ }^{\mathrm{b}} p<0.05$ vs. the TMG group, ${ }^{\mathrm{c}} p<0.05$ vs. the TSLG group, ${ }^{\mathrm{d}} p<0.05$ vs. the TSMG group, and ${ }^{\mathrm{e}} p<0.05$ vs. the TSHG group.

and GPx (Figure 11(c)) and reduced the MDA level (Figure 11(d), $p<0.05)$. FMT of Saikosaponin A-treated rats suppressed oxidative stress in the rats with SAP.

Anti-inflammatory analysis showed that serum levels of TNF- $\alpha$ (Figure 11(e)), IL-1 $\beta$ (Figure 11(f)), and IL-6 (Figure 11(g)) were lowest in the TCG group and significantly increased in the TMG group while the IL-10 level was highest in the TCG group and lowest in the TMG group (Figure 11(h), $p<0.05)$. FMT of Saikosaponin A-treated rats reduced the serum levels of serum levels of TNF- $\alpha$ (Figure 11(e)), IL-1 $\beta$ (Figure 11(f)), and IL-6 (Figure 11(g)) and increased the IL-10 level (Figure 11(h), $p<0.05$ ). The levels of serum CRP (Figure 11(i)) and serum PCT (Figure 11(j)) significantly increased in the TMG group when compared with the TCG group $(p<0.05)$. The results suggested that FMT of SAP model rats significantly increased the levels of serum CRP and PCT. On the other hand, FMT of Saikosaponin A-treated rats reduced the level of serum CRP (Figure 11(i)) and serum PCT (Figure 11(j), $p<0.05)$. FMT of Saikosaponin A-treated rats increased anti-inflammatory properties in the rats with SAP.

3.9. FMT of Saikosaponin A-Treated Rats Increased the Relative mRNA Levels of Antioxidant Signaling Protein. The relative mRNA levels of Keap1 (Figure 12(a)), Nrf2 (Figure 12(b)), and ARE (Figure 12(c)) was obviously decreased in the TMG group when compared with those in the TCG group $(p<0.05)$. However, FMT of Saikosaponin A-treated rats increased the relative mRNA levels of Keap1 (Figure 12(a)), Nrf2 (Figure 12(b)), and ARE (Figure 12(c)). The experiment result demonstrated that FMT of Saikosaponin A-treated rats ameliorated the SAP by increasing the relative mRNA levels of antioxidant signaling molecules.

3.10. FMT of Saikosaponin A-Treated Rats Increased the Expression of Antioxidant Signaling Protein. Western blot analysis showed that the expression levels of Keap1 (Figure 13(a)), Nrf2 (Figure 13(b)), and ARE (Figure 13(c)) obviously decreased in the TMG group when compared with the TCG group $(p<0.05)$. However, FMT of Saikosaponin A-treated rats increased the expression levels of Keap1 (Figure 13(a)), Nrf2 (Figure 13(b)), and ARE (Figure 13(c)). The experiment result demonstrated that FMT of Saikosaponin A-treated rats ameliorated the SAP by increasing the expression of antioxidant signaling protein.

3.11. FMT of Saikosaponin A-Treated Rats Improved Gut Microbiota Composition. The abundance of Lactobacillus species was highest in the TCG, and lowest in the TMG group (Figure 14(a)). FMT of Saikosaponin A-treated rats increased the relative abundance of Lactobacillus species (Figure 14(a)). The abundance of Prevotella species was similar between the TCG and TMG groups (Figure 14(a)). 


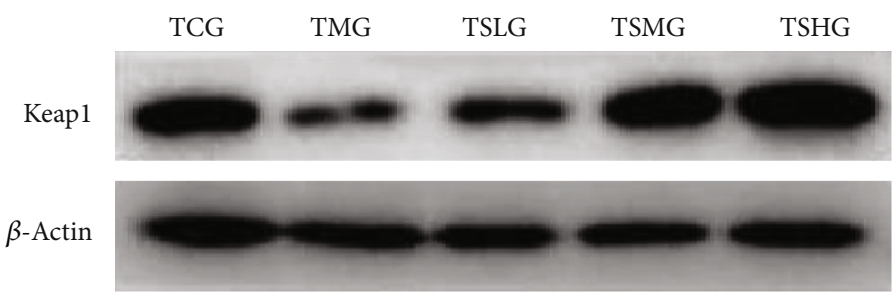

(a)

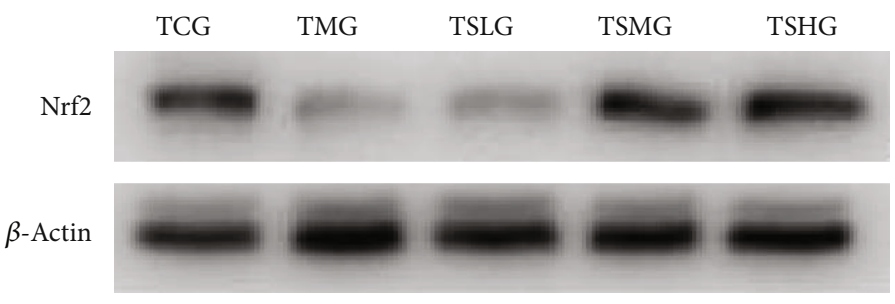

(b)

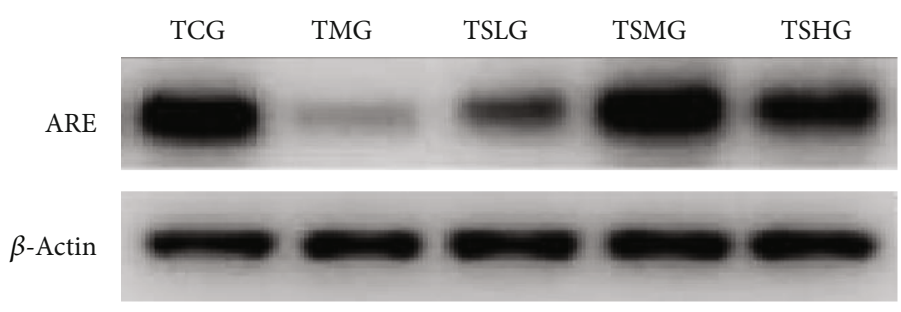

(c)
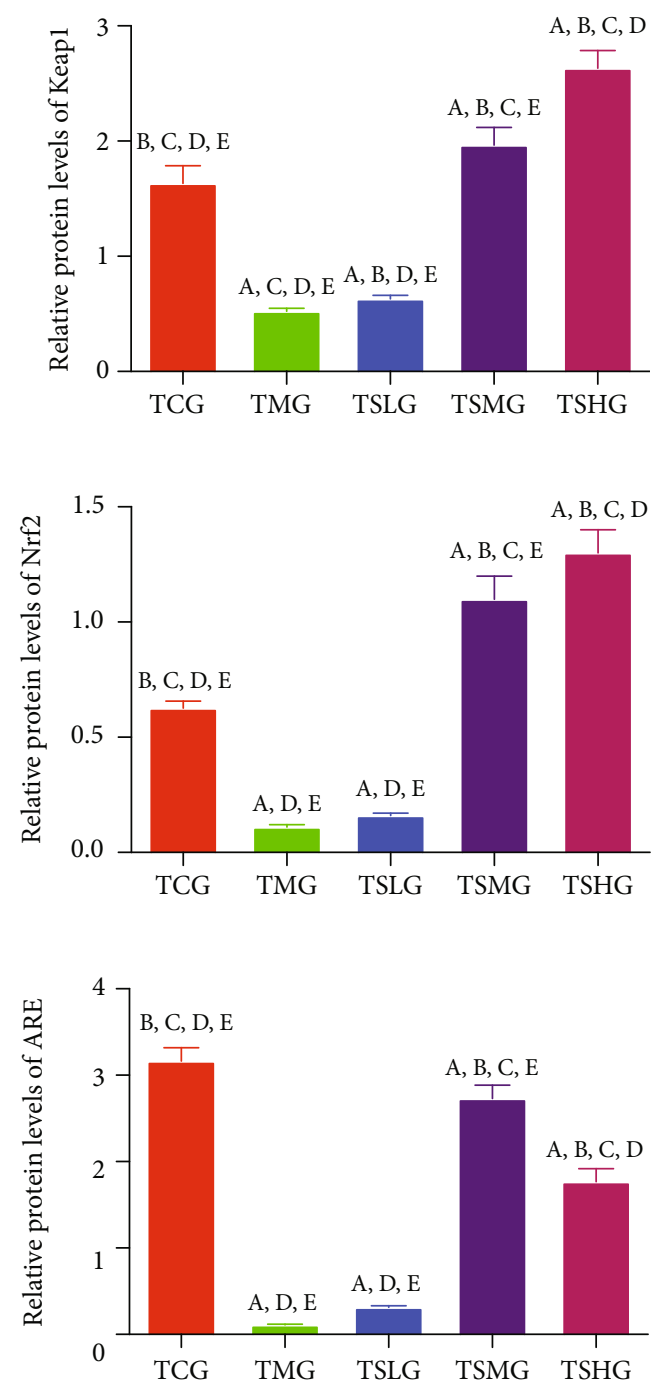

Figure 13: Western blot analysis of the effects of FMT of Saikosaponin A on the expression of antioxidant signaling proteins. (a) Kelch-like ECH-associated protein 1 (Keap1). (b) Nuclear factor erythroid 2-related factor 2 (NRF2). (c) Antioxidant response element (ARE). Data were presented as means \pm S.D. (standard deviation) and $n=8$ for each group. ${ }^{\mathrm{a}} p<0.05$ vs. the TCG group, ${ }^{\mathrm{b}} p<0.05$ vs. the TMG group, ${ }^{c} p<0.05$ vs. the TSLG group, ${ }^{\mathrm{d}} p<0.05$ vs. the TSMG group, and ${ }^{\mathrm{e}} p<0.05$ vs. the TSHG group.

FMT of Saikosaponin A-treated rats increased the relative abundance of Prevotella species in a dose-dependent way (Figure 14(a)). Heatmap analysis showed the similar results as the barplots (Figure 14(b)). Therefore, FMT of Saikosaponin A-treated rats improved the gut microbiota by increasing the proportion of Lactobacillus and Prevotella species (Figure 14). The results indicated that FMT of Saikosaponin A-treated rats improved gut microbiota in the SAP model.

\section{Discussion}

SAP lesions are linked with sepsis, infected pancreatic necrosis, and multiorgan failure [47]. SAP is a relapsing complication of digestive system and can lead to chronic inflammatory disease [48]. Many drugs are used for the treatment of SAP but most of them have unwanted adverse effects [49]. The present work showed that Saikosaponin A exerted protective effects against SAP risk by reducing pathological scores (Figure 4) and increasing antioxidant and antiinflammation (Figure 5) properties. We next examined the effects of Saikosaponin A on the expression of antioxidant signaling molecules. Saikosaponin A administration significantly improved the expression of Keap1, Nrf2, and ARE (Figures 7 and 8). Furthermore, Saikosaponin A intervention also increased anti-inflammatory capacity by reducing the levels of IL-6, IL- $1 \beta$, and TNF- $\alpha$ and increased the level of IL-10 (Figure 5). The results were consistent with the previous reports that Saikosaponin A treatment reduced the IL-6, IL- $1 \beta$, and TNF- $\alpha$ levels [50] and increased IL-10 level $[50,51]$. Saikosaponin A treatment also reduced the levels of main inflammatory factors of SAP, CRP, and PCT (Figure 5), but the related report was not found yet. These results suggest that Saikosaponin A is a potential drug in the prevention of SAP progression. 


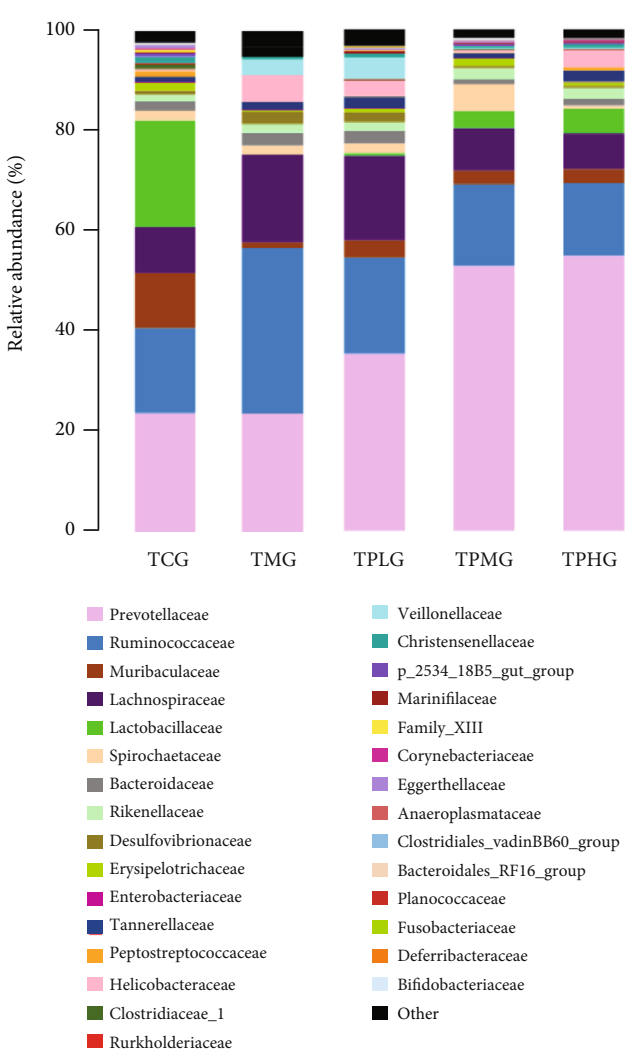

(a)

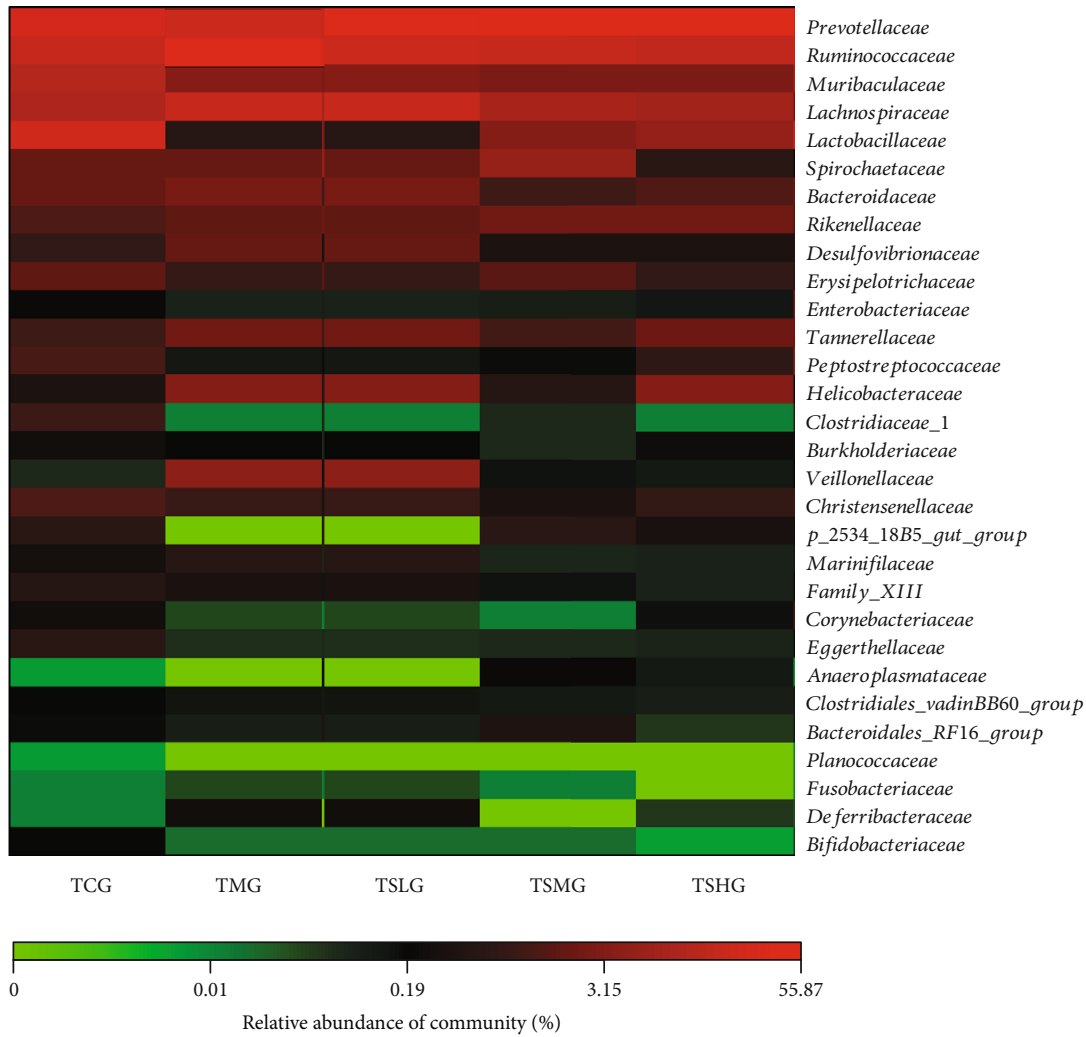

(b)

FIgURE 14: The effects of FMT on composition of gut microbiota among different groups. (a) The proportion of gut microbiota. (b) Heatmap analysis of gut microbiota changes from different FMT. 


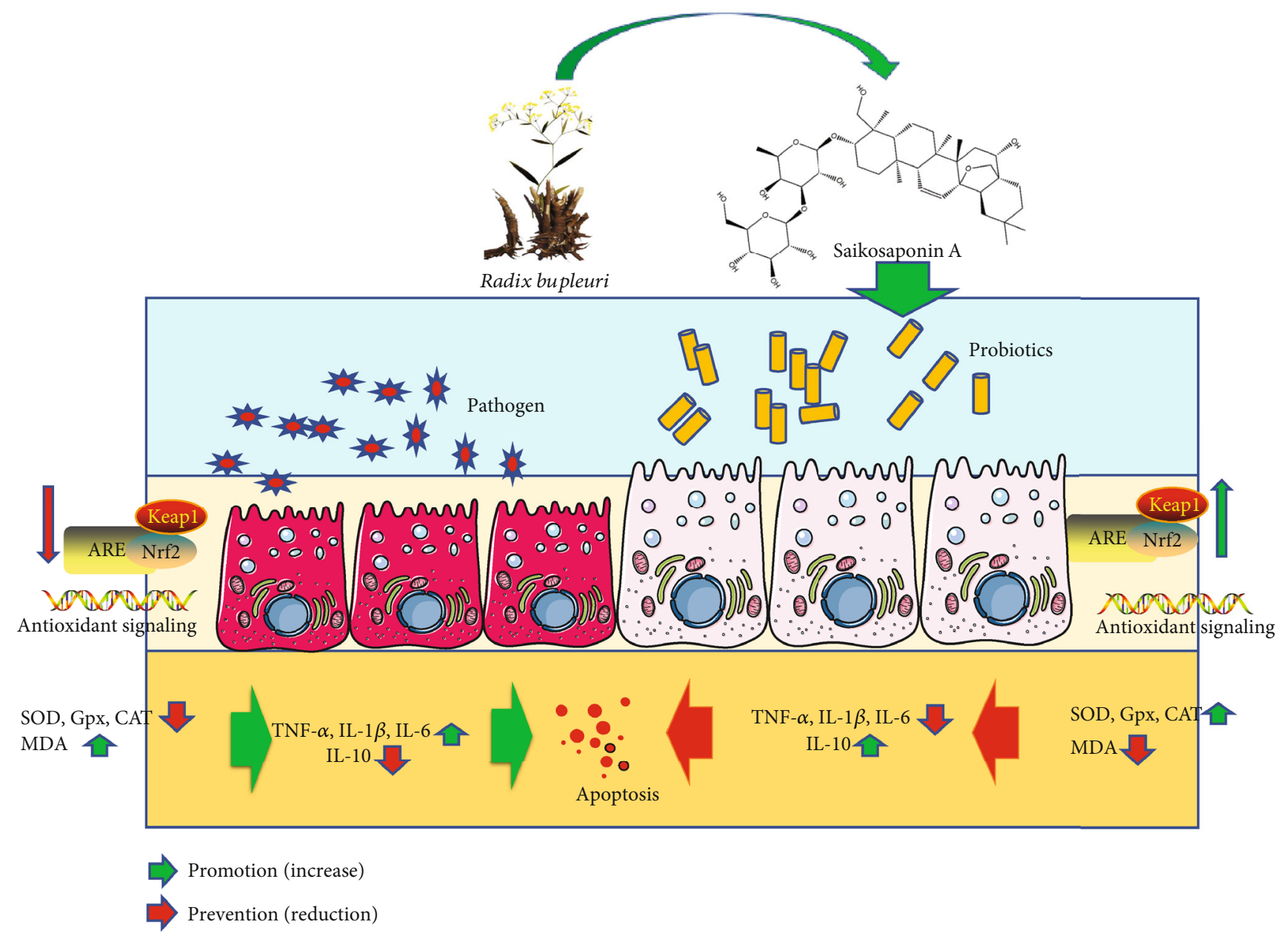

FIgURE 15: Saikosaponin A from Radix bupleuri prevented severe acute pancreatitis. Saikosaponin A intervention improved gut microbiota composition, which reduces inflammation responses and improves antioxidant properties via Kelch-like ECH-associated protein 1-nuclear factor erythroid 2-related factor 2-antioxidant response element (Keap1-Nrf2-ARE) signaling.

Saikosaponin A treatment improved antioxidant capacity by increasing the serum levels of SOD, CAT, and GPx and reducing the MDA level. Free radicals and reactive oxygen species (ROS) and reactive nitrogen species (RNS) contribute to many pancreatitis processes by inducing oxidative stress and oxidative damage $[29,52,53]$. The increase in the ROS level induces DNA damage and potential cytotoxicity [54]. SOD shows its antioxidant role by directly scavenging excess intracellular free radicals and reducing MDA level and enhancing total antioxidant capacity (T-AOC) [55]. Decreased antioxidant SOD activity will induce the increase in the levels of oxidative stress biomarkers (MDA) in the SAP model [56]. GPx is a selenium-dependent enzyme that prevents intracellular hydrogen peroxide and lipid peroxides [57]. CAT widely exists in mammalian cells and shows the protection against from ROS, which is produced through the decomposition of $\mathrm{H}_{2} \mathrm{O}_{2}$ [58]. Serum activity of GPx and SOD is closely associated with the removal of ROS [59]. SOD, CAT, and GPx are indispensable in the defense against oxidative species into bloodstream, especially super oxide anion radical $\left(\mathrm{O}_{2} \cdot-\right)$, which is continuously produced in human body metabolism via the mitochondrial energy production pathway [60].
The ROS superoxide anion radical is mainly generated by NADPH oxidase (NOX) in the SAP model and usually converted into $\mathrm{H}_{2} \mathrm{O}_{2}$ with the participation of SOD, as well as NO generated by inducible nitric oxide synthase. The latter ROS generated from XO plays a crucial role in SAP injury [29]. The previous reports showed that Saikosaponin A reduced the expression of NOX [61]. The extracts with Saikosaponin A inhibited the superoxide anion formation by $\mathrm{XO}[62]$.

Nrf2-ARE pathway also attenuates oxidative stressinduced DNA damage in pancreatic beta cells [63]. Keap1Nrf2-ARE are important antioxidant signaling and exerts protective function against SAP. The expression of Keap1Nrf2-ARE was significantly downregulated in SAP rats. The change was inhibited via Saikosaponin A administration. Many antioxidant enzyme systems are expressed by activating the Keap1-Nrf2-ARE signaling pathway [64]. These results indicate that Saikosaponin A may exert an important protective effect on pancreas integrity by affecting the expression of Keap1-Nrf2-ARE antioxidant signaling.

On the other hand, the treatment of Saikosaponin A treatment improved gut microbiota composition (Figure 8). 
Saikosaponin A may show its function via the improvement of gut microbiota composition, and the results of FMT further confirmed such a proposal. To explore the effects of FMT on pancreatitis, histology analysis was also performed. FMT of Saikosaponin A-treated rats increased colon length and reduced DAI and pathological scores. FMT of Saikosaponin A-treated rats also increased antioxidant and antiinflammation properties (Figure 11). Meanwhile, the FMT of Saikosaponin A-treated rats increased the expression of antioxidant signaling molecules of Keap1-Nrf2-ARE (Figure 13).

Additionally, Saikosaponin A intervention improved gut microbiota and showed significant increases in the probiotics, including Lactobacillus and Prevotella species (Figure 8). Similarly, FMT of Saikosaponin A-treated rats also improved gut microbiota. FMT-treated samples showed significant increases in the probiotics, including Lactobacillus and Prevotella species (Figure 14). Lactobacillus species suppressed and repair E. coli-impaired SAP by increasing the expression and distribution of antioxidant proteins and can be served as an essential food additive to solve health complications. Lactobacillus as a new generation of probiotics plays an important role in maintaining intestinal epithelial homeostasis and exerting healthpromoting function [65]. Prevotella species may exert their function by fermenting carbohydrate and releasing shortchain fatty acid (SCFA) to protect pancreas integrity [66]. Notably, Prevotella copri is potential harmful bacterium which can be inhibited by lactobacillus bacteria [67]. Surprisingly, the abundance of Lactobacillus and Prevotella species was increased after supplementation with Saikosaponin A or gavage with FMT. These results suggest that Saikosaponin A-induced gut microbiota reduces SAP by reducing inflammatory responses and improving antioxidant signaling through gut microbiota (Figure 15).

There were some limitations in the present study. The redox homeostasis was only analyzed in the blood sample although it is more important to measure the redox homeostasis in pancreatic tissues for better understanding the effects of Saikosaponin A on the SAP risk. The serum concentrations of ROS and RNS were not measured although ROS and RNS levels are closely associated with oxidative stress. Albumin is a very abundant and important circulating antioxidant with ligand binding and strong DPPH $(1,1-$ diphenyl-2-picrylhydrazyl) radical scavenging activity properties $[68,69]$. However, serum albumin was not measured in the present experiment. Furthermore, only the activities of selected redox biomarkers were evaluated without considering other critical redox protein, DNA oxidation products, ROS production rate, etc. The effects of FMT on healthy rats were not evaluated. Finally, the rat models could not adequately imitate the clinical scenarios of human SAP. The effects of Saikosaponin A treatment on microbiota-depleted rats were not explored in the present work, and thus, the function of Saikosaponin A may be affected by the preexisting gut microbiota. Saikosaponin A intervention may induce the production of an antimicrobial peptide (AMP), which can change the composition of gut microbiota by reducing the bacterial translocation. The decrease in the AMP levels will result in the dysbiosis of gut microbiota in the AP model [70]. The FMT may contain such AMP and affect the distribution of gut microbiota. Therefore, the effect of FMT in normal animals should be studied if AMP can be identified and used to treat AP. Further work is needed to address these important issues.

In conclusion, this study indicates that administration of Saikosaponin A intervention attenuates SAP lesions. Saikosaponin A treatment not only increased antioxidant and antiinflammatory properties but also improved SAP lesions in the rat model. These findings indicate that Saikosaponin Ainduced gut microbiota changes may have a potential protective effect on SAP and are useful in the prevention of the inflammatory disease as SAP.

\section{Data Availability}

All data are available from the corresponding author (Yanfang Jiang, Email: jiangyfjl@126.com) upon reasonable request.

\section{Conflicts of Interest}

There is no conflict of interest.

\section{Authors' Contributions}

J. L., J. H., and S. W. conceived and designed the experiments. J. L., S. W., and L. Q. performed the experiments and analyzed data. L. Q. and Y. J. wrote the paper. Jing Li and Jinfeng Han contributed equally to this work.

\section{Acknowledgments}

The project was supported by the creative project of Jilin University. We are very grateful to Professor Jie Liu for his technical support and experimental design.

\section{References}

[1] L. Medina Andrade, A. Delgado, L. Perez Corona, O. Moreno, and D. Rodríguez, "Acute pancreatitis, actualization and evidence based management," Archives of Clinical Gastroenterology, vol. 3, no. 1, pp. 1-8, 2017.

[2] W. Deng, A. Abliz, S. Xu et al., "Severity of pancreatitisassociated intestinal mucosal barrier injury is reduced following treatment with the NADPH oxidase inhibitor apocynin," Molecular Medicine Reports, vol. 14, no. 4, pp. 3525-3534, 2016.

[3] M. Schietroma, B. Pessia, F. Carlei, P. Mariani, F. Sista, and G. Amicucci, "Intestinal permeability and systemic endotoxemia in patients with acute pancreatitis," Annali Italiani di Chirurgia, vol. 87, no. 1, pp. 138-144, 2016.

[4] J. Samanta, S. Singh, S. Arora et al., "Cytokine profile in prediction of acute lung injury in patients with acute pancreatitis," Pancreatology, vol. 18, no. 8, pp. 878-884, 2018.

[5] C. Skouras, Z. A. Davis, J. Sharkey et al., "Lung ultrasonography as a direct measure of evolving respiratory dysfunction and disease severity in patients with acute pancreatitis," $H P B$, vol. 18, no. 2, pp. 159-169, 2016.

[6] N. R. Thiruvengadam, K. A. Forde, V. Chandrasekhara et al., "Tacrolimus and indomethacin are safe and effective at 
reducing pancreatitis after endoscopic retrograde cholangiopancreatography in patients who have undergone liver transplantation," Clinical Gastroenterology and Hepatology, vol. 18, pp. 1224-1232.e1, 2020.

[7] X. Chai, H. B. Huang, G. Feng et al., "Baseline serum cystatin C is a potential predictor for acute kidney injury in patients with acute pancreatitis," Disease Markers, vol. 2018, Article ID 8431219, 7 pages, 2018.

[8] T. P. Fitzgibbons, J. Paolino, J. C. Dagorn, and T. E. Meyer, "Usefulness of pancreatitis-associated protein, a novel biomarker, to predict severity of disease in ambulatory patients with heart failure," The American Journal of Cardiology, vol. 113, no. 1, pp. 123-126, 2014.

[9] Y. Zhou, L. Wang, X. Huang, H. Li, and Y. Xiong, “Add-on effect of crude rhubarb to somatostatin for acute pancreatitis: a meta-analysis of randomized controlled trials," Journal of Ethnopharmacology, vol. 194, pp. 495-505, 2016.

[10] J. Li, S. Zhang, R. Zhou, J. Zhang, and Z. F. Li, “Perspectives of traditional Chinese medicine in pancreas protection for acute pancreatitis," World Journal of Gastroenterology, vol. 23, no. 20, pp. 3615-3623, 2017.

[11] H. Wang, H. Zheng, Z. Zhao, and P. Chen, "Effect of Chaihu Shihuang soup on blood serum level TNF, IL-6 and IL-10 of severe acute pancratitis (SAP)," Zhongguo Zhong Yao Za Zhi, vol. 34, no. 12, pp. 1582-1584, 2009.

[12] Y. Wang, L. Zhao, X. Han et al., "Saikosaponin A inhibits triple-negative breast cancer growth and metastasis through downregulation of CXCR4," Frontiers in Oncology, vol. 9, article 1487, 2019.

[13] J. Guo, F. Zhang, J. Gao et al., "Proteomics-based screening of the target proteins associated with antidepressant-like effect and mechanism of Saikosaponin A," Journal of Cellular and Molecular Medicine, vol. 24, no. 1, pp. 174-188, 2020.

[14] P. Feng, Y. Xu, B. Tong et al., "Saikosaponin a attenuates hyperlipidemic pancreatitis in rats via the PPAR- $\gamma / \mathrm{NF}-\mathrm{iB}$ signaling pathway," Experimental and Therapeutic Medicine, vol. 19, no. 2, pp. 1203-1212, 2020.

[15] V. Cifarelli, S. Ivanov, Y. Xie et al., "CD36 deficiency impairs the small intestinal barrier and induces subclinical inflammation in mice," Cellular and Molecular Gastroenterology and Hepatology, vol. 3, no. 1, pp. 82-98, 2017.

[16] X. Y. Li, C. He, Y. Zhu, and N. H. Lu, "Role of gut microbiota on intestinal barrier function in acute pancreatitis," World Journal of Gastroenterology, vol. 26, no. 18, pp. 2187-2193, 2020.

[17] X. Li, C. He, N. Li et al., "The interplay between the gut microbiota and NLRP3 activation affects the severity of acute pancreatitis in mice," Gut Microbes, vol. 11, no. 6, pp. 17741789, 2020.

[18] G. Zhang, R. Cui, Y. Kang et al., "Testosterone propionate activated the Nrf2-ARE pathway in ageing rats and ameliorated the age-related changes in liver," Scientific Reports, vol. 9, no. 1, article 18619, 2019.

[19] M. C. Lu, J. A. Ji, Z. Y. Jiang, and Q. D. You, “The Keap1-Nrf2ARE pathway as a potential preventive and therapeutic target: an update," Medicinal Research Reviews, vol. 36, no. 5, pp. 924-963, 2016.

[20] H. H. Hagar, S. A. Almubrik, N. M. Attia, and S. N. Aljasser, "Mesna alleviates cerulein-induced acute pancreatitis by inhibiting the inflammatory response and oxidative stress in experimental rats," Digestive Diseases and Sciences, 2020.
[21] R. Ma, F. Yuan, S. Wang, Y. Liu, T. Fan, and F. Wang, "Calycosin alleviates cerulein-induced acute pancreatitis by inhibiting the inflammatory response and oxidative stress via the p38 MAPK and NF- $\kappa \mathrm{B}$ signal pathways in mice," Biomedicine \& Pharmacotherapy, vol. 105, pp. 599-605, 2018.

[22] G. Marek, M. Ściskalska, Z. Grzebieniak, and H. Milnerowicz, "Decreases in paraoxonase-1 activities promote a proinflammatory effect of lipids peroxidation products in nonsmoking and smoking patients with acute pancreatitis," International Journal of Medical Sciences, vol. 15, no. 14, pp. 1619-1630, 2018.

[23] S. Cao, Y. Bian, X. Zhou et al., "A small-molecule activator of mitochondrial aldehyde dehydrogenase 2 reduces the severity of cerulein-induced acute pancreatitis," Biochemical and Biophysical Research Communications, vol. 522, no. 2, pp. 518$524,2020$.

[24] M. Sajadian, M. Hashemi, S. Salimi, and A. Nakhaee, "The effect of experimental thyroid dysfunction on markers of oxidative stress in rat pancreas," Drug Development Research, vol. 77, no. 4, pp. 199-205, 2016.

[25] N. E. El-Ashmawy, N. F. Khedr, H. A. El-Bahrawy, and O. B. Hamada, "Suppression of inducible nitric oxide synthase and tumor necrosis factor-alpha level by lycopene is comparable to methylprednisolone in acute pancreatitis," Digestive and Liver Disease, vol. 50, no. 6, pp. 601-607, 2018.

[26] E. M. El Morsy and M. A. E. Ahmed, "Carvedilol attenuates 1arginine induced acute pancreatitis in rats through modulation of oxidative stress and inflammatory mediators," ChemicoBiological Interactions, vol. 327, article 109181, 2020.

[27] X. Piao, B. Liu, X. Sui et al., "Picroside II improves severe acute pancreatitis-induced intestinal barrier injury by inactivating oxidative and inflammatory TLR4-dependent PI3K/AKT/ $\mathrm{NF}-\kappa \mathrm{B}$ signaling and improving gut microbiota," Oxidative Medicine and Cellular Longevity, vol. 2020, Article ID 3589497, 12 pages, 2020.

[28] G. G. Kojayan, R. F. Alizadeh, S. Li, and H. Ichii, "Reducing pancreatic fibrosis using antioxidant therapy targeting Nrf2 antioxidant pathway: a possible treatment for chronic pancreatitis," Pancreas, vol. 48, no. 10, pp. 1259-1262, 2019.

[29] M. Maciejczyk, A. Skutnik-Radziszewska, I. Zieniewska et al., "Antioxidant defense, oxidative modification, and salivary gland function in an early phase of cerulein pancreatitis," Oxidative Medicine and Cellular Longevity, vol. 2019, Article ID 8403578, 14 pages, 2019.

[30] Z. Y. Xiao, X. R. Li, W. Y. Shao, and S. H. Wu, "Purification and concentration of the Total saikosaponins extracted from radix bupleuriusing foam fractionation," Separation Science and Technology, vol. 49, no. 3, pp. 469-475, 2014.

[31] H. Aho, T. Nevalainen, R. Lindberg, and A. Aho, "Experimental pancreatitis in the rat: the role of phospholipase $\mathrm{A}$ in sodium taurocholate-induced acute haemorrhagic pancreatitis," Scandinavian Journal of Gastroenterology, vol. 15, no. 8, pp. 1027-1031, 2010.

[32] R. Mukherjee, O. A. Mareninova, I. V. Odinokova et al., "Mechanism of mitochondrial permeability transition pore induction and damage in the pancreas: inhibition prevents acute pancreatitis by protecting production of ATP," Gut, vol. 65, no. 8, pp. 1333-1346, 2016.

[33] D. Liu, G. Song, Z. Ma et al., "Resveratrol improves the therapeutic efficacy of bone marrow-derived mesenchymal stem cells in rats with severe acute pancreatitis," International Immunopharmacology, vol. 80, article 106128, 2020. 
[34] X. Zhou, H. Cheng, D. Xu et al., "Attenuation of neuropathic pain by saikosaponin a in a rat model of chronic constriction injury," Neurochemical Research, vol. 39, no. 11, pp. 21362142, 2014.

[35] H. B. Meng, J. Gong, B. Zhou, J. Hua, L. Yao, and Z. S. Song, "Therapeutic effect of human umbilical cord-derived mesenchymal stem cells in rat severe acute pancreatitis," International Journal of Clinical and Experimental Pathology, vol. 6, no. 12, pp. 2703-2712, 2013.

[36] J. Ali, A. U. Khan, F. A. Shah et al., "Mucoprotective effects of Saikosaponin-A in 5-fluorouracil-induced intestinal mucositis in mice model," Life Sciences, vol. 239, article 116888, 2019.

[37] W.-W. Wang, Y. Zhang, X.-B. Huang, N. You, L. Zheng, and J. Li, "Fecal microbiota transplantation prevents hepatic encephalopathy in rats with carbon tetrachloride-induced acute hepatic dysfunction," World Journal of Gastroenterology, vol. 23, no. 38, pp. 6983-6994, 2017.

[38] P. Shen, Z. Zhang, K. Zhu et al., "Evodiamine prevents dextran sulfate sodium-induced murine experimental colitis via the regulation of NF- $\kappa \mathrm{B}$ and NLRP3 inflammasome," Biomedicine \& Pharmacotherapy, vol. 110, pp. 786-795, 2019.

[39] X. Fu, P. Li, W. Yin et al., "Overexpression of Nrf2 protects against lipopolysaccharide and cerulein-induced pancreatitis in vitro and in vivo," Pancreas, vol. 49, no. 3, pp. 420-428, 2020.

[40] X. Song, J. Li, Y. Wang et al., "Clematichinenoside AR ameliorated spontaneous colitis in Il-10-/- mice associated with improving the intestinal barrier function and abnormal immune responses," Life Sciences, vol. 239, article 117021, 2019.

[41] F. A, N. K, and L. D, "DSC examination of intestinal tissue following cold preservation," Thermochimica Acta, vol. 497, pp. 41-45, 2010.

[42] F. Zhao, Z. Huang, G. Zhou, H. Li, X. Xu, and C. Li, "Dietary proteins rapidly altered the microbial composition in rat caecum," Current Microbiology, vol. 74, no. 12, pp. 1447-1452, 2017.

[43] T. Strate, O. Mann, H. Kleinhans et al., "Systemic intravenous infusion of bovine hemoglobin significantly reduces microcirculatory dysfunction in experimentally induced pancreatitis in the rat," Annals of Surgery, vol. 238, no. 5, pp. 765-771, 2003.

[44] R. K. McNamara, R. Jandacek, T. Rider, P. Tso, A. ColeStrauss, and J. W. Lipton, "Atypical antipsychotic medications increase postprandial triglyceride and glucose levels in male rats: relationship with stearoyl-CoA desaturase activity," Schizophrenia Research, vol. 129, no. 1, pp. 66-73, 2011.

[45] C. D’Ambrosio, T. Bowman, and V. Mohsenin, "Quality of life in patients with obstructive sleep apnea: effect of nasal continuous positive airway pressure-a prospective study," Chest, vol. 115, no. 1, pp. 123-129, 1999.

[46] Y. Gao, J. Li, S. Chu et al., "Ginsenoside Rg1 protects mice against streptozotocin-induced type 1 diabetic by modulating the NLRP3 and Keap1/Nrf2/HO-1 pathways," European Journal of Pharmacology, vol. 866, article 172801, 2020.

[47] I. K. Uchendu, C. E. Agu, O. C. Orji, M. C. Offor, and T. F. Nwosu, "Review on diagis of acute pancreatitis," Journal of Medical \& Allied Sciences, vol. 7, no. 2, p. 76, 2017.

[48] A. Garcia Garcia de Paredes, E. Rodriguez de Santiago, C. Rodriguez-Escaja et al., "Idiopathic acute pancreatitis in patients with inflammatory bowel disease: a multicenter cohort study," Pancreatology, vol. 20, no. 3, pp. 331-337, 2020.
[49] H. Vargas-Robles, K. F. Castro-Ochoa, A. F. Citalán-Madrid, and M. Schnoor, "Beneficial effects of nutritional supplements on intestinal epithelial barrier functions in experimental colitis modelsin vivo," World Journal of Gastroenterology, vol. 25, no. 30, pp. 4181-4198, 2019.

[50] J. Zhu, C. Luo, P. Wang, Q. He, J. Zhou, and H. Peng, "Saikosaponin A mediates the inflammatory response by inhibiting the MAPK and NF- $\kappa$ B pathways in LPS-stimulated RAW 264.7 cells," Experimental and Therapeutic Medicine, vol. 5, no. 5, pp. 1345-1350, 2013.

[51] M. Q. Huang, X. Y. Cao, X. Y. Chen et al., "Saikosaponin a increases interleukin-10 expression and inhibits scar formation after sciatic nerve injury," Neural Regeneration Research, vol. 13, no. 9, pp. 1650-1656, 2018.

[52] L. Wen, T. A. Javed, A. K. Dobbs et al., "The protective effects of calcineurin on pancreatitis in mice depend on the cellular source," Gastroenterology, vol. 159, no. 3, pp. 1036-1050.e8, 2020.

[53] J. E. Fishman, G. Levy, V. Alli, X. Zheng, D. J. Mole, and E. A. Deitch, "The intestinal mucus layer is a critical component of the gut barrier that is damaged during acute pancreatitis," Shock, vol. 42, no. 3, pp. 264-270, 2014.

[54] M. Cal, I. Matyjaszczyk, I. Litwin et al., "The anticancer drug 3Bromopyruvate induces DNA damage potentially through reactive oxygen species in yeast and in human cancer cells," Cell, vol. 9, no. 5, p. 1161, 2020.

[55] J. Pan, L. Li, L. Liang et al., "Cytoprotective effects of cellpermeable bifunctional antioxidant enzyme, GST-TAT-SOD, against cisplatin-induced cell damage," Oxidative Medicine and Cellular Longevity, vol. 2017, Article ID 9530791, 7 pages, 2017.

[56] A. M. Abdel-Aziz, R. A. Rifaai, and S. A. Abdel-Gaber, "Possible mechanisms mediating the protective effect of cilostazol in $\mathrm{L}$-arginine induced acute pancreatitis in rats: role of cGMP, cAMP, and HO-1," Naunyn-Schmiedeberg's Archives of Pharmacology, vol. 393, no. 10, pp. 1859-1870, 2020.

[57] J.-Q. Huang, J.-C. Zhou, Y.-Y. Wu, F.-Z. Ren, and X. G. Lei, "Role of glutathione peroxidase 1 in glucose and lipid metabolism-related diseases," Free Radical Biology \& Medicine, vol. 127, pp. 108-115, 2018.

[58] A. Reale, R. G. Ianniello, F. Ciocia et al., "Effect of respirative and catalase-positive Lactobacillus casei adjuncts on the production and quality of Cheddar-type cheese," International Dairy Journal, vol. 63, pp. 78-87, 2016.

[59] J. Dworzański, M. Strycharz-Dudziak, E. Kliszczewska et al., "Glutathione peroxidase (GPx) and superoxide dismutase (SOD) activity in patients with diabetes mellitus type 2 infected with Epstein-Barr virus," PLoS One, vol. 15, no. 3, article e0230374, 2020.

[60] O. Ighodaro and O. Akinloye, "First line defence antioxidantssuperoxide dismutase (SOD), catalase (CAT) and glutathione peroxidase (GPX): their fundamental role in the entire antioxidant defence grid," Alexandria Journal of Medicine, vol. 54, no. 4, pp. 287-293, 2018.

[61] J. Li, S. Biswas, Y. Niu et al., "P23 Saikosaponin a ameliorate learning and memory impairment via anti-inflammation effect in an AD mouse model," Biochemical Pharmacology, vol. 139, p. 132, 2017.

[62] C.-T. Liu, C.-Y. Wu, Y.-M. Weng, and C.-Y. Tseng, "Ultrasound-assisted extraction methodology as a tool to improve the antioxidant properties of herbal drug Xiao-Chia-HuTang," Journal of Ethnopharmacology, vol. 99, no. 2, pp. 293300, 2005. 
[63] P. Vanitha, S. Senthilkumar, S. Dornadula, S. Anandhakumar, P. Rajaguru, and K. M. Ramkumar, "Morin activates the Nrf2ARE pathway and reduces oxidative stress-induced DNA damage in pancreatic beta cells," European Journal of Pharmacology, vol. 801, pp. 9-18, 2017.

[64] M.-C. Lu, J. Zhao, Y.-T. Liu et al., "CPUY192018, a potent inhibitor of the Keap1-Nrf2 protein-protein interaction, alleviates renal inflammation in mice by restricting oxidative stress and NF- $\kappa$ B activation," Redox Biology, vol. 26, article 101266, 2019.

[65] Q. Liu, Z. Yu, F. Tian et al., "Surface components and metabolites of probiotics for regulation of intestinal epithelial barrier," Microbial Cell Factories, vol. 19, no. 1, article 23, 2020.

[66] C. le Bourgot, S. Ferret-Bernard, E. Apper et al., "Perinatal short-chain fructooligosaccharides program intestinal microbiota and improve enteroinsular axis function and inflammatory status in high-fat diet-fed adult pigs," The FASEB Journal, vol. 33, no. 1, pp. 301-313, 2018.

[67] W. Ou, H. Hu, P. Yang et al., "Dietary daidzein improved intestinal health of juvenile turbot in terms of intestinal mucosal barrier function and intestinal microbiota," Fish \& Shellfish Immunology, vol. 94, pp. 132-141, 2019.

[68] S. Minic, D. Stanic-Vucinic, M. Radomirovic et al., "Characterization and effects of binding of food-derived bioactive phycocyanobilin to bovine serum albumin," Food Chemistry, vol. 239, pp. 1090-1099, 2018.

[69] S. Wu, X. Wang, Y. Bao et al., "Molecular insight on the binding of monascin to bovine serum albumin (BSA) and its effect on antioxidant characteristics of monascin," Food Chemistry, vol. 315, article 126228, 2020.

[70] J. Chen, C. Huang, J. Wang et al., "Dysbiosis of intestinal microbiota and decrease in paneth cell antimicrobial peptide level during acute necrotizing pancreatitis in rats," PLoS One, vol. 12, no. 4, article e0176583, 2017. 